\title{
Key polynomials in dimension 2
}

\author{
W. Mahboub \\ Lebanese University, Lebanon. \\ M. Spivakovsky \\ Institut de Mathématiques de Toulouse \\ UMR 5219 du CNRS, \\ Université Paul Sabatier \\ 118, route de Narbonne \\ 31062 Toulouse cedex 9, France. \\ email: mark.spivakovsky@math.univ-toulouse.fr
}

January 30, 2019 


\section{Introduction}

Throughout this paper all the rings considered will be commutative with 1 .

Let $(R, \mathfrak{m}, k)$ be a regular local ring of dimension 2 and $F$ the field of fractions of $R$. Consider the poset $(\mathcal{V}, \leq)$ of normalized valuations of $F$ centered at $R$ (see 92 ).

In 4] C. Favre and M. Jonsson prove that $(\mathcal{V}, \leq)$ has the structure of a parametrized, rooted, non-metric tree when $R=\mathbb{C}[[x, y]]$, the ring of formal power series over the field of complex numbers. The proof of C. Favre and M. Jonsson is based on associating to each valuation in $\mathcal{V}$ a set of key polynomials, a concept introduced by S. MacLane in [9] and [10]. Below we will refer to this set as a complete set of key polynomials (see 84 for its definition and porperties).

In [5] A. Granja generalizes this result to the case when $R$ is any two-dimensional regular

local ring. A. Granja gives a proof based on associating to each valuation in $\mathcal{V}$ a sequence of point blowing ups.

In this paper we give a new proof of A. Granja's result when $R$ is any two-dimensional regular local ring, using appropriate complete sequences of key polynomials, based on the work of M. Vaquié [17] for valuations of arbitrary rank, and the work of F. J. Herrera Govantes, W. Mahboub, M. A. Olalla Acosta and M. Spivakovsky ([6, [7]) for valuations of rank 1 over fields of arbitrary characteristic.

We use the notion of key polynomials introduced in [3] and [14]. We give a simple construction of a complete set of key polynomials associated to a valuation of the field $k(x, y)$ where $k$ is the residue field of $R$ and $x, y$ are independent variables. For explicit constructions of key polynomials on particular cases, see [15], 4], 8].

We start by stating in 92 the basic facts related to valuations needed in this paper. Then we establish, in 93 , a natural order-preserving bijection between valuations of $F$ centered at $R$ and valuations of $k(x, y)$ centered at $k[x, y]_{(x, y)}$. This is the content of Theorem 3.12 , It consists of describing a one-to-one correspondence between the set of valuations centered at $R$ and the set of simple sequences of local point blowing ups (see Corollary 3.11).

In 4 we give the definition of key polynomials. We state the needed facts about key polynomials and construct a complete set of key polynomials associated to a valuation $\nu$ of $k(x, y)$. This is our main tool for the proof of Theorem 6.4. We also define invariants of valuations centered in regular two-dimensional local rings.

Then we consider two comparable valuations, $\mu \leq \nu$, and study the structure of their key polynomials sets and the relation between the invariants of those valuations. This is done in $\$ 5$, Using this comparison, we prove that the infimum of any two elements of $\mathcal{V}$ exists (Theorem 5.8) and that any increasing sequence in $\mathcal{V}$ has a majorant in $\mathcal{V}$ (Theorem 5.91). We note that a more general version of the latter result — one for rings of arbitrary dimension — is given in Lemma 3.9 (i) of [13].

Finally, in 96 we prove the main theorem of this paper, Theorem6.4. This Theorem asserts that $\mathcal{V}$ has a tree structure.

We thank the referee for a very careful reading of the paper and for numerous useful 
comments that helped improve the exposition.

\section{Basics}

Let $R$ be a regular noetherian local ring of dimension 2 . Denote by $\mathfrak{m}$ its maximal ideal and let $F$ be the quotient field of $R$.

A valuation of $F$ is a function $\nu: F \longrightarrow \overline{\mathbb{R}}=\mathbb{R} \cup\{\infty\}$ such that for all $f, g \in F$ :

$\left(V_{1}\right) \nu(f+g) \geq \inf (\nu(f), \nu(g))$,

$\left(V_{2}\right) \nu(f \cdot g)=\nu(f)+\nu(g)$.

It is an easy exercise to check that if $\nu$ is not constant, then axiom $\left(V_{2}\right)$ implies $\left(V_{3}\right) \nu(1)=0$.

Let $\Gamma$ be a totally ordered abelian group. A Krull valuation of $F$ is a function

$$
\nu: F \longrightarrow \Gamma \cup\{\infty\}
$$

satisfying $\left(V_{1}\right),\left(V_{2}\right)$ and $\left(V_{3}\right)$ such that $\nu^{-1}(\infty)=0$.

If $\nu$ is a valuation or a Krull valuation of $F$, we say that $\nu$ is centered at $R$ if $\nu$ is non-negative on $R$ and strictly positive on $\mathfrak{m}$. We say that $\nu$ is proper if $\nu(F \backslash\{0\}) \neq\{0\}$ and $\nu(\mathfrak{m}) \neq\{\infty\}$.

If $\nu$ and $\nu^{\prime}$ are two valuations of $F$, then we say that $\nu$ and $\nu^{\prime}$ are equivalent, and write $\nu \sim \nu^{\prime}$, if there exists a non-zero real number $c$ such that for all $f \in F$ we have $\nu(f)=c \nu^{\prime}(f)$.

Let $\mathcal{V}=\{\nu \mid \nu$ proper valuation centered at $R\} / \sim$. When working with an element of $\mathcal{V}$, we will tacitly fix a valuation representing it, so in practice we will work with valuations instead of classes of valuations. We will consider only normalized valuations, in the sense that $\nu(\mathfrak{m}):=\inf \{\nu(f) \mid f \in \mathfrak{m}\}=1$. Indeed, we can represent any element $\nu$ of $\mathcal{V}$ by a uniquely determined normalized valuation after multiplying all the values by $\frac{1}{\nu(\mathfrak{m})}$.

For an element $\nu \in \mathcal{V}$ we will denote by $\Gamma_{\nu}$ the augmented value group of $\nu$, that is, $\Gamma_{\nu}:=\nu(F) \subset \overline{\mathbb{R}}$

If $\nu$ is a valuation (resp. a Krull valuation), the set

$$
R_{\nu}:=\{f \in F \mid \nu(f) \geq 0\}
$$

is a local ring called the valuation ring associated to $\nu$, with maximal ideal

$$
\mathfrak{m}_{\nu}:=\{f \in F \mid \nu(f)>0\} .
$$

The rank of $\nu$, denoted by $\operatorname{rk}(\nu)$, is the Krull dimension of $R_{\nu}$. In our situation $\operatorname{rk}(\nu)$ is at most 2 by Abhyankar's inequality.

Remark 2.1. We have $\operatorname{rk}(\nu)=1$ if and only if $\nu(F \backslash\{0\}) \subset \mathbb{R}$ (resp. the group $\nu(F \backslash\{0\})$ can be embedded into the additive group $\mathbb{R}$ of real numbers). 
If $S$ is a ring contatined in $R_{\nu}$, the center of $\nu$ in $S$ is the prime ideal $\mathfrak{m}:=\mathfrak{m}_{\nu} \cap S$. In this situation we also say that $\nu$ is centered at $\mathfrak{m}$. If $(S, \mathfrak{m})$ is a local domain, we will sometimes say that $\nu$ is centered at $(S, \mathfrak{m})$.

Definition 2.2. For two local rings $\left(R_{1}, \mathfrak{m}_{1}\right)$ and $\left(R_{2}, \mathfrak{m}_{2}\right)$, we say that $R_{2}$ dominates $R_{1}$ if $R_{1} \subset R_{2}$ and $\mathfrak{m}_{1}=R_{1} \cap \mathfrak{m}_{2}$. If, in addition, $R_{1}$ and $R_{2}$ are domains with the same field of fractions, we will say that $R_{2}$ birationally dominates $R_{1}$

Notation. In the above situation we will write $\left(R_{1}, \mathfrak{m}_{1}\right)<\left(R_{2}, \mathfrak{m}_{2}\right)$ or simply $R_{1}<R_{2}$.

Remark 2.3. Let $(S, \mathfrak{m})$ be a local domain, contained in $F$. A valuation $\nu$ of $F$ is centered at $\mathfrak{m}$ if and only if we have $(S, \mathfrak{m})<\left(R_{\nu}, \mathfrak{m}_{\nu}\right)$.

Remark 2.4. (1) The valuation $\nu$ is uniquely determined by its valuation ring $R_{\nu}$. For a proof, see [16], Proposition 1.4.

(2) Consider a local domain $(R, \mathfrak{m})$ with field of fractions $F$. The following conditions are equivalent:

(a) $(R, \mathfrak{m})$ is of the form $\left(R_{\nu}, \mathfrak{m}_{\nu}\right)$ for some valuation $\nu$ of $F$

(b) for every $f \in F$ either $f \in R_{\nu}$ or $f^{-1} \in R_{\nu}$ (or both)

(c) the ring $(R, \mathfrak{m})$ is maximal with respect to the relation of birational domination.

For a proof, see [16], Proposition 1.4 and [2], Chap. 6, $\S 2, \mathrm{n}^{0} 2$, Théorème 1, page 85.

Below we reprove the equivalence $(\mathrm{a}) \Longleftrightarrow(\mathrm{c})$ in the special case when $R$ is a 2 -dimensional regular local ring.

We will use the following partial order on the set of valuations of the field $F$, centered at $R$ :

For two valuations $\mu$ and $\mu^{\prime}$ centered at $R$, we will say that $\mu \leq \mu^{\prime}$ if $\mu(f) \leq \mu^{\prime}(f)$ for all $f \in R$.

We denote by $\tilde{\nu}_{\mathfrak{m}}$ the multiplicity valuation, that is, $\tilde{\nu}_{\mathfrak{m}}(f):=\max \left\{i \mid f \in \mathfrak{m}^{i}\right\}$ for all $f \in R$. We note that $\tilde{\nu}_{\mathfrak{m}}$ is the smallest element of $\mathcal{V}$. We say that the multiplicity of $f$ at $\mathfrak{m}$ is $\tilde{\nu}_{\mathfrak{m}}(f)$.

If $\nu$ is a valuation centered at $R$ then $\nu$ determines a Krull valuation $\nu^{\prime}$, centered at $R$. Furthermore, $\operatorname{rk}\left(\nu^{\prime}\right)=2$ if and only if $\nu^{-1}(\infty) \neq\{0\}$. Indeed, if $\nu^{-1}(\infty)=\{0\}$, then $\nu=\nu^{\prime}$ is a Krull valuation of rank 1. Otherwise, if $\nu^{-1}(\infty) \neq\{0\}$, then $\nu^{-1}(\infty)$ is a principal prime ideal of $R$ generated by an irreducible element $f \in R$. For each $g \in R-\{0\}$, write $g=f^{s} h$, where $f$ does not divide $h$ (that is, $\nu(h)<\infty)$. Define $\nu^{\prime}(g)=(s, \nu(h)) \in \mathbb{Z} \oplus \mathbb{R}$. For $G=\frac{g_{1}}{g_{2}} \in F-\{0\}$, put $\nu(G)=\nu\left(g_{1}\right)-\nu\left(g_{2}\right)$. It is clear that $\nu^{\prime}$ determines a Krull valuation on $F$, centered at $R$.

Conversely, if $\nu^{\prime}$ is a Krull valuation of $F$ centered at $R$ then $\nu^{\prime}$ determines a valuation on $R$. Indeed, let $\mathfrak{m}_{\nu^{\prime}}$ be the maximal ideal of $R_{\nu^{\prime}}$. If $\operatorname{rk}\left(\nu^{\prime}\right)=1$, put $\nu=\nu^{\prime}$. Otherwise, let $\Gamma_{1}$ be the isolated subgroup of $\Gamma$ of rank 1 (that is, the smallest non-zero isolated subgroup of $\Gamma$ ). Let $P^{\prime}$ be the prime ideal of $R_{\nu^{\prime}}$ associated to $\Gamma_{1}$ :

$$
P^{\prime}=\left\{f \in R_{\nu^{\prime}} \mid \nu(f) \in \Gamma \backslash \Gamma_{1}\right\} .
$$

Let $P=P^{\prime} \cap R$. For each $f \in R$, if $f \in P$, put $\nu(f)=\infty$. Otherwise, put

$$
\nu(f)=\nu^{\prime}(f) \in \Gamma_{1} .
$$


Then $\nu$ is a valuation centered at $R$.

For an element $\beta \in \Gamma_{\nu}$, let $\mathbf{P}_{\beta}:=\{f \in F \mid \nu(f) \geq \beta\}, \mathbf{P}_{\beta+}:=\{f \in F \mid \nu(f)>\beta\}$. Let

$\operatorname{gr}_{\nu} F=\bigoplus_{\beta \in \Gamma_{1}} \frac{\mathbf{P}_{\beta}}{\mathbf{P}_{\beta+}}$. For an element $\beta \in \Gamma_{1}$ and an element $f \in F$ such that $\nu(f)=\beta$, we will denote by $\operatorname{in}_{\nu} f$ the natural image of $f$ in $\frac{\mathbf{P}_{\beta}}{\mathbf{P}_{\beta+}}$.

\section{$3 \quad$ Valuations and blowing ups}

The aim of this section is to describe a natural order-preserving bijection between valuations of $F$ centered at $R$ and valuations of $k(x, y)$ centered at $k[x, y]_{(x, y)}$.

Remark 3.1. Throughout the paper we will commit the following abuse of notation. We will use the letters $x, y$ to denote both the generators of the field $k(x, y)$ over $k$ and a regular system of parameters of $R$. Since in each case we will specify clearly with which ring we are working, this should cause no confusion.

A simple sequence $\pi^{*}$ of local point blowings up of Spec $R$ is a sequence of the form

$$
(R, \mathfrak{m}) \stackrel{\pi_{1}}{\longrightarrow}\left(R_{1}, \mathfrak{m}_{1}\right) \stackrel{\pi_{2}}{\longrightarrow} \ldots \stackrel{\pi_{i}}{\longrightarrow}\left(R_{i}, \mathfrak{m}_{i}\right) \stackrel{\pi_{i+1}}{\longrightarrow} \ldots
$$

where $\pi_{i}$ is given by considering the blowing up Spec $R_{i} \stackrel{\pi_{i}^{*}}{\longrightarrow}$ Spec $R_{i-1}$ along $\mathfrak{m}_{i-1}$, picking a point $\xi_{i} \in \pi_{i}^{*-1}\left(\mathfrak{m}_{i-1}\right)$ and putting $R_{i}:=\mathcal{O}_{X_{i}, \xi_{i}}$. Let $\Pi(R)$ denote the set of all the simple sequences (finite or infinite) of local point blowings up of Spec $R$. Fix an element $f \in R \backslash\{0\}$. Let $\mu$ denote the multiplicity of $f$ at $\mathfrak{m}$. Assume that

$$
f \notin\left(x, y^{\mu+1}\right) .
$$

Definition 3.2. A monomial ideal in a regular local ring $A$, with regular system of parameters $\left(u_{1}, \ldots, u_{s}\right)$ is an ideal in $A$ generated by monomials in $\left(u_{1}, \ldots, u_{s}\right)$.

Let $I(x, y, f)$ denote the smallest monomial ideal containing $f$. (3.2) is equivalent to saying that $y^{\mu} \in I(x, y, f)$.

Let $e(x, y, f):=\min \left\{\frac{\alpha}{\mu-\beta} \mid x^{\alpha} y^{\beta} \in I(x, y, f), \beta<\mu\right\} \in \frac{1}{\mu !} \mathbb{Z} \cup\{\infty\}$, where we adopt the convention that the minimum of the empty set is infinity.

Definition 3.3. The first characteristic exponent of $f$ at $\mathfrak{m}$ is the supremum of $e(x, y, f)$, where $(x, y)$ runs over all the regular systems of parameters of $R$ satisfying (3.2).

Fix a real number $e$. For a real number $\xi$, let $I_{\xi}$ denote the monomial ideal of $R$ generated by all the monomials $x^{\alpha} y^{\beta}$ such that $\alpha+e \beta \geq \xi$.

Definition 3.4. The monomial valuation $\nu_{x, y, e}$ of $R$, associated to the data $(x, y, e)$ is the valuation defned by $\nu_{x, y, e}(g)=\max \left\{\xi \in \mathbb{R} \mid g \in I_{\xi}\right\}$.

Let $R^{*}$ denote the set of units in $R$.

Proposition 3.5. Let $e_{0}$ be the first characteristic exponent of $f$ at $\mathfrak{m}$. Let $e=e(x, y, f)$.

The following conditions are equivalent:

1. $e<e_{0}$,

2. $e$ is an integer, and there exists a regular system of parameters of the form $\left(y-u x^{e}, x\right)$, with $u$ a unit of $R$, satisfying $e(x, y, f)<e\left(x, y-u x^{e}, f\right)$. 
3. $e$ is an integer and $\operatorname{in}_{\nu_{x, y, e}} f$ is the $\mu$-th power of a linear form in $\operatorname{in}_{\nu_{x, y},} y$ and $\operatorname{in}_{\nu_{x, y, e}} x^{e}$; more specifically, there exist $c, d \in k$ such that

$$
\operatorname{in}_{\nu_{x, y, e}} f=\left(\operatorname{in}_{\nu_{x, y, e}} y-c \operatorname{in}_{\nu_{x, y}, e} x^{e}\right)^{\mu} .
$$

Proof. 1) $\Longrightarrow 2$ ) Since $e_{0}>e$, there exists a change of coordinates

$$
\begin{aligned}
& x_{1}=a_{1} x+a_{2} y^{l_{1}} \\
& y_{1}=b_{1} y+b_{2} x^{l_{2}}
\end{aligned}
$$

with $a_{1}, a_{2}, b_{1}, b_{2} \in R^{*}$ such that

$$
e_{1}:=e\left(x_{1}, y_{1}, f\right)>e(x, y, f)
$$

Replacing $x$ by $a_{1}^{-1}\left(x_{1}-a_{2} y^{l_{1}}\right)$ does not change $e$, therefore, we may assume that $x_{1}=x$. Since $b_{1} \in R^{*}$, we may assume that $b_{1}=1$. Now we will prove that $e=l_{2}$.

Write

$$
f=\sum_{i+j e_{1} \geq \mu e_{1}} a_{i j} x^{i} y_{1}^{j}=\sum_{i+j e_{1} \geq \mu e_{1}} a_{i j} x^{i}\left(y+b_{2} x^{l_{2}}\right)^{j},
$$

where $a_{0 \mu} \in R^{*}$. Consider a monomial of the form $a_{i j} x^{i} y_{1}^{j}$ with

$$
i+j e_{1} \geq \mu e_{1} .
$$

The element $x^{i} y_{1}^{j}$ belongs to the monomial ideal of $R$ generated by the set

$$
\left\{x^{i+s l_{2}} y^{j-s} \mid s \in\{0, \ldots, j\}\right\}
$$

Let

$$
e^{\prime}=\min \left\{e_{1}, l_{2}\right\}
$$

Let us prove that $e=e^{\prime}$. Indeed, if $i, j$ satislfy (3.8) then

$$
i \geq(\mu-j) e_{1}
$$

If $s \in\{0, \ldots, j\}$ then, since $l_{2} \geq e^{\prime}$, we obtain

$$
i+s l_{2} \geq(\mu-j) e_{1}+s l_{2} \geq(\mu-(j-s)) e^{\prime} .
$$

Thus $e^{\prime} \leq e$. Combined with (3.6) and (3.9), this proves that

$$
e^{\prime}=l_{2}<e_{1}
$$

Combining (3.12) with (3.11), we obtain

$$
i+s l_{2} \geq(\mu-(j-s)) l_{2}
$$

and the inequality is strict unless $i=0$ and $j=\mu$. Thus

$$
e=\min \left\{\frac{\alpha}{\mu-\beta} \mid x^{\alpha} y^{\beta} \in I(x, y, f), \beta<\mu\right\}=\min \left\{\frac{s l_{2}}{\mu-(\mu-s)} \mid s \in\{1, \ldots \mu\}\right\}=l_{2} .
$$


Therefore, $e \in \mathbb{N}$ and $y_{1}=y+u x^{e}$ with $u \in R^{*}$ satisfies the conclusion of (2).

$$
\begin{aligned}
& \text { 2) } \Longrightarrow 3) \text { Let } e^{\prime}=e\left(x, y-u x^{e}, f\right) \text { and write } f=\left(y-u x^{e}\right)^{\mu}+\sum_{i+j e^{\prime} \geq \mu e^{\prime}} a_{i j} x^{i}\left(y-u x^{e}\right)^{j} \text {. } \\
& (i, j) \neq(0, \mu)
\end{aligned}
$$

To prove 3) it is sufficient to prove that $\nu_{x, y, e}\left(\sum_{\substack{i+j e^{\prime} \geq \mu e^{\prime} \\(i, j) \neq(0, \mu)}} a_{i j} x^{i}\left(y-u x^{e}\right)^{j}\right)>\mu e$. Now, $\sum_{e^{\prime} \geq \mu e^{\prime}} a_{i j} x^{i}\left(y-u x^{e}\right)^{j}$ is contained in the monomial ideal (with respect to $\left.(x, y)\right)$, gen$(i, j) \neq(0, \mu)$

erated by monomials of the form $\left(\begin{array}{l}j \\ s\end{array}\right) a_{i j} x^{i} y^{j-s}\left(u x^{e}\right)^{s}$ with $0 \leq s \leq j, i+e^{\prime} j \geq \mu e^{\prime}$ and if $j=\mu$ then $i>0$. Now we have to prove that the quantity $q=i+s e+(j-s) e$ is strictly greater than $\mu e$. We have $q=i+j e$. If $j=\mu$, then $i>0$ and $q>\mu e$. If $j>\mu$ then $q>\mu e$. If $j<\mu$, then $q=i+j e \geq e^{\prime}(\mu-j)+j e=\mu e-\mu e+e^{\prime}(\mu-j)+j e=\mu e+(\mu-j)\left(e^{\prime}-e\right)>\mu e$. This completes the proof of $(3)$.

$3) \Longrightarrow 1$ ) Choose $u \in R^{*}$ such that the natural image of $u$ in $k$ is $c$. We have

$$
f=\left(y-u x^{e}\right)^{\mu}+\sum_{i, j} a_{i j} x^{i} y^{j}
$$

with $\nu_{x, y, e}\left(\sum a_{i j} x^{i} y^{j}\right)>\mu e$, that is $i+j e>\mu e$ for all the $(i, j)$ appearing in the sum. Put $y_{1}=y-u x^{e}$. We will prove that $e^{\prime}=e\left(x, y_{1}, f\right)>e$.

We have $f=y_{1}^{\mu}+\sum_{i, j} \sum_{s=0}^{j}\left(\begin{array}{l}j \\ s\end{array}\right) a_{i j} x^{i+e s} y^{j-s}$ with $i+j e>\mu e$ for each $(i, j)$ in the sum.

Now we have $e^{\prime} \geq \frac{i+e s}{\mu-(j-s)}>\frac{(\mu-j) e+e s}{\mu-(j-s)}=e$ whenever $(j-s)<\mu$.

Remark 3.6. Let $e$ denote the first characteristic exponent of $f$. If $R$ is quasi-excellent, $f$ is reduced and $\mu \geq 2$, we have

$$
e \in \frac{1}{\mu !} \mathbb{N}
$$

that is, $1 \leq e<\infty$. Since in this paper we work with arbitrary regular two-dimensional local rings and not just the quasi-excellent ones, we will not use this fact in the sequel.

Fix a simple sequence of point blowings up as in (3.1). Let $\mu_{i}(f)$ and $e_{i}(f)$ denote, respectively, the multiplicity and the first characteristic exponent of the strict transform of $f$ in $R_{i}$.

Lemma 3.7. At least one of the following conditions holds:

(1) $\left(\mu_{i+1}, e_{i+1}\right)<_{l e x}\left(\mu_{i}, e_{i}\right)$

(2) $e_{i}(f)=\infty$.

Proof. To simplify the notation, we will consider the case when $i=0$, so that $R_{0}=R$. Assume that $e_{i}(f) \neq \infty$. Let $f_{1}$ be the strict transform of $f$ in $R_{1}$. We will follow the notation of ([20], 
Appendix 5, pagse 365-367). Namely, let $\bar{g}$ denote the directional form of the local blowing up $\pi_{1}$ and $\bar{f}$ the natural image of $f$ in $\operatorname{gr}_{\mathfrak{m}} R$.

Let $\mu=\mu_{0}$ denote the multiplicity of $f$ at $\mathfrak{m}$.

Since $\bar{f}$ is a homogeneous polynomial of degree $\mu$, the greatest power of $\bar{g}$ that could divide $\bar{f}$ is $\bar{g}^{\mu}$. If $\bar{g}^{\mu}$ does not divide $\bar{f}$, then by ([20], Appendix 5, page 367, Proposition 2), we have $\mu_{1}<\mu$ and (1) of the Lemma holds.

Assume that $\bar{g}^{\mu} \mid \bar{f}$. Then $\operatorname{deg} \bar{g}=1$ and there exists a regular system of parameters $(x, y)$ such that $\bar{g}=\bar{y}$ and

$$
f=y^{\mu}+\sum_{i+j>\mu} a_{i j} x^{i} y^{j}
$$

where the $a_{i j}$ are units of $R$. Let $e=e_{0}$ denote the first characteristic exponent of $f$ and choose $(x, y)$ in such a way that $e=e(x, y, f)$. Write $f=y^{\mu}+\sum_{i+j e=\mu e} a_{i j} x^{i} y^{j}+\sum_{i+j e>\mu e} a_{i j} x^{i} y^{j}$. $(i, j) \neq(0, \mu)$

Since $e<\infty$, there exists $(i, j) \neq(0, \mu)$, with $i+j e=\mu e$.

$$
\text { Now } f_{1}=y_{1}^{\mu}+\sum_{\substack{i+j e=\mu e \\ \\(i, j) \neq(0, \mu)}} a_{i j} x_{1}^{i+j-\mu} y_{1}^{j}+\sum_{i+j e>\mu e} a_{i j} x_{1}^{i+j-\mu} y_{1}^{j} .
$$

Note that for each $(i, j)$ with $i+j e=\mu e$ we have $(i+j-\mu)+j(e-1)=\mu(e-1)$ and for each $(i, j)$ with $i+j e>\mu e$ we have $(i+j-\mu)+j(e-1)>\mu(e-1)$.

If $s>e-1$ then for $(i, j)$ with $i+j e=\mu e$ we have $(i+j-\mu)=(\mu-j)(e-1)<(\mu-j) s$, hence $(i+j-\mu)+j s<\mu s$.

If $e-1<1$ then $\mu_{1}<\mu$. Otherwise, if $e-1 \geq 1$, the above considerations prove that $e\left(x_{1}, y_{1}, f_{1}\right)=e-1$.

By Proposition 3.5, $\operatorname{in}_{\nu_{x, y, e}} f$ is not a $\mu$-th power of a linear form in $\operatorname{in}_{\nu_{x, y, e}} y$ and $\operatorname{in}_{\nu_{x, y, e}} x^{e}$. Hence $\operatorname{in}_{\nu_{x_{1}, y_{1}, e-1}} f_{1}$ is not a $\mu$-th power of a linear form in $\operatorname{in}_{\nu_{x_{1}, y_{1}, e-1}} y_{1}$ and $\operatorname{in}_{\nu_{x_{1}, y_{1}, e-1}} x_{1}^{e}$. Therefore $e_{1}=e\left(x_{1}, y_{1}, f_{1}\right)=e-1<e$. In all the cases (1) of the Lemma holds.

Lemma 3.8. Let $\pi^{*}$ be an infinite sequence of local blowings up belonging to $\Pi(R)$. Write $\pi^{*}$ as in (3.1). Take an element $f \in R \backslash\{0\}$.

(1) If $f$ is a unit in $R_{j_{0}}$ for some $j_{0}$, then $f$ is a unit in $R_{j}$ for all $j \in \mathbb{N}$.

(2) At least one of the following conditions holds:

(a) there exists $i \in \mathbb{N}$ such that $f=x_{i}^{s} y_{i}^{t} u$ where $x_{i}$ and $y_{i}$ are regular parameters of $R_{i}, s$ and $t$ are natural numbers and $u$ is a unit of $R_{i}$

(b) there exists $i_{0} \in \mathbb{N}$ such that $e_{i}(f)=\infty$ for all $i \geq i_{0}$.

Proof. 1. This follows directly from the fact that for all $j \in \mathbb{N}$, we have $\mathfrak{m}_{j}=R_{j} \cap \mathfrak{m}_{j+1}$.

2. First note that if $x_{i}$ and $y_{i}$ are regular parameters of $R_{i}$, then either $x_{i}=x_{i+1} y_{i+1}$ and $y_{i}=y_{i+1}$ or $x_{i}=x_{i+1}$ and $y_{i}=\frac{y_{i}}{x_{i}} x_{i+1}$, with $\frac{y_{i}}{x_{i}}$ is either a unit in $R_{i+1}$ or equal to $y_{i+1}$.

Assume that condition (b) does not hold. From Lemma 3.7 we deduce that for each $j^{\prime} \in \mathbb{N}$ there exists $j>j^{\prime}$ with $\mu_{j}<\mu_{j^{\prime}}$. Hence there exists $j \in \mathbb{N}$ with $f_{j} \in R^{*}$. Now by definition of $f_{j}, f_{j-1}=x^{\mu_{j-1}} f_{j}$, therefore, using the paragraph above and induction, we get the result. 
For an element $\pi^{*} \in \Pi(R)$ we denote $\bar{R}=\bigcup_{i} R_{i}$. The ring $\bar{R}$ is an integral domain with quotient field $F$, dominating $R$ and $R_{i}$ for each $i \in i^{i} \mathbb{N}$.

Proposition 3.9. Assume that there exists $f \in R$, satisfying condition (2)(b) in Lemma 3.8. Then there exists a unique Krull valuation $\nu$ on $F$ such that $R_{\nu}$ dominates $\bar{R}$.

Moreover, we have:

1. $\operatorname{rk} \nu=2$.

2. The set of elements in $R$ satisfying condition (2)(b) in Lemma 3.8 is a prime ideal generated by an irreducible element $g$.

3. let $g_{i}$ denote the strict transform of $g$ in $R_{i}$. Then $\nu$ is the composition of the g-adic valuation of $F$ with the unique rank one Krull valuation, centered in the one-dimensional local rings $\frac{R_{i}}{\left(f_{i}\right)}$ for each $i \in \mathbb{N}$.

Proof. Since $\bar{R}$ is an integral domain with field of fractions $F$, there exists a valuation ring $R_{\nu}$ dominating $\bar{R}$. Let $\Gamma$ denote the value group of this valuation. Now to prove the uniqueness of $\nu$ it is sufficient to prove the conditions (1), (2) and (3).

1. Let $f_{i}$ denote the strict transform of $f$ in $R_{i}$. We have $\mu_{i}=\mu_{i_{0}}>0$ for all $i \geq i_{0}$. Let $\left(x_{i_{0}}, y_{i_{0}}\right)$ be a regular system of parameters of $R_{i_{0}}$ such that $f_{i_{0}}=y_{i_{0}}^{\mu_{i_{0}}}+$ terms of higher degree. Since $\mu_{i}=\mu_{i_{0}}$, we have $x_{i_{0}}=x_{i_{0}+1}$. Now $f_{i_{0}+1}=y_{i_{0}}^{\mu_{i_{0}}}+$ terms of higher or equal degree. Repeating the same reasoning, we see that for each $i \geq i_{0}$ we have $x_{i}=x_{i_{0}}$ and $f_{i}=x_{i+1}^{\mu_{i+1}} f_{i+1}=x_{i_{0}}^{\mu_{i_{0}}} f_{i+1}$. Thus $f_{i_{0}}=x_{i_{0}}^{\left(i-i_{0}\right) \mu_{i_{0}}} f_{i}$ for each $i>i_{0}$. Since $f_{i} \in R_{\nu}$ for all $i$, we have $\nu\left(f_{i}\right)>0$ for all $i$. Hence $\nu\left(f_{i_{0}}\right)>i \mu_{i_{0}} \nu\left(x_{i_{0}}\right)$ for all $i$, so $\nu\left(f_{i_{0}}\right)$ cannot belong to a subgroup of $\Gamma$ of rank 1. Therefore $r k(\nu)=2$.

2. Let $\Gamma_{1}$ denote the isolated subgroup of $\Gamma$ of rank 1 (that is, the unique proper non-trivial subgroup of $\Gamma$ ). Let $P^{\prime}$ be the prime ideal of $R_{\nu}$ associated to $\Gamma_{1}$. Let $P=P^{\prime} \cap R$. Then $P$ is a prime ideal of height 1 in $R$, therefore it is generated by an irreducible element $g$. Now $f=h g^{n}$ with $h \notin P$, hence $\nu(h) \in \Gamma_{1}$. Therefore, by the proof of (1), there exists $i$ such that $h_{i}$ the strict transform of $h$ in $R_{i}$ is a unit. Now the strict transform of $f$ in $R_{i}$ is $h_{i} \cdot g_{i}^{n}$. Therefore $g$ must also satisfy condition (2)(b) in Lemma 3.8. An element of $R$ satisfies condition (2)(b) in Lemma 3.8 if and only if it belongs to $P$.

3. This is a direct consequence of (1) and (2).

Proposition 3.10. Let $\pi^{*}$ be an element of $\Pi(R)$ and write $\pi^{*}$ as in (3.1). Suppose that $R$ does not contain an element $f$ satisfying condition (2) (b) of Lemma 3.8. The following statements hold. $i \in \mathbb{N}$.

(1) The ring $\bar{R}$ is a valuation ring with field of fractions $F$, dominating $R$ and $R_{i}$ for each

(2) Conversely, let $R_{\mu}$ be a valuation ring with field of fractions $F$, dominating $R$ and $R_{i}$ for each $i \in \mathbb{N}$. Then $R_{\mu}=\bar{R}$.

In other words, the simple blowing up sequence $\pi^{*}$ and the valuation $\mu$ determine each other uniquely; they are equivalent sets of data. 
Proof. (1) Since $R<R_{i}<R_{j}$ for all natural numbers $i \leq j, \bar{R}$ is a domain with quotient field $F$, dominating $R$ and $R_{i}$ for each $i \in \mathbb{N}$. First, consider the case when the sequence

$$
\pi^{*}:(R, \mathfrak{m}) \stackrel{\pi_{1}}{\longrightarrow}\left(R_{1}, \mathfrak{m}_{1}\right) \stackrel{\pi_{2}}{\longrightarrow} \ldots \stackrel{\pi_{n}}{\longrightarrow}\left(R_{n}, \mathfrak{m}_{n}\right)
$$

is finite. Then $\bar{R}=R_{n}$. By definition, $\mathfrak{m}_{n}$ is principal and $\left(R_{n}, \mathfrak{m}_{n}\right)$ is a discrete valuation ring.

Next, assume that $\pi^{*}$ is infinite. To prove that $\bar{R}$ is a valuation ring, consider an element $f \in F^{*}$, and write $f=\frac{f_{1}}{f_{2}}$ where $f_{1}, f_{2} \in R \backslash\{0\}$.

By Lemma 3.8 there exists $i \in \mathbb{N}$ such that $f_{1}=x_{i}^{s_{1}} y_{i}^{t_{1}} u_{1}$ and $f_{2}=x_{i}^{s_{2}} y_{i}^{t_{2}} u_{2}$, where $x_{i}$ and $y_{i}$ are local parameters in $R_{i}, s_{1}, t_{1}, s_{2}$ and $t_{2}$ are natural numbers and $u_{1}, u_{2}$ are units in $R_{i}$. Hence

$$
f=x_{i}^{s} y_{i}^{t} u
$$

where $s$ and $t$ are integers (not necessarily positive) and $u$ is a unit in $R_{i}$. If both $s$ and $t$ are non-negative then $f \in R_{i} \subset \bar{R}$, as desired. If both $s$ and $t$ are non-postive then $\frac{1}{f} \in R_{i} \subset \bar{R}$, as desired. Otherwise assume, without loss of generality, that $s>0$ and $t<0$. Now after another blowing up, we have the following three possibilities:

$$
\begin{aligned}
& f=x_{i+1}^{s} y_{i+1}^{s+t} u \quad \text { or } \\
& f=x_{i+1}^{s+t} y_{i+1}^{t} u \quad \text { or } \\
& f=y_{i+1}^{s+t} v
\end{aligned}
$$

where $x_{i+1}$ and $y_{i+1}$ are local parameters in $R_{i+1}$ and, in the last equation, $v$ is a unit in $R_{i+1}$. If (3.18) holds and $s+t \geq 0$ then $f \in R_{i+1} \subset \bar{R}$. If (3.18) holds and $s+t \leq 0$ then $f^{-1} \in R_{i+1} \subset \bar{R}$. According to Remark 2.4 (2), $\bar{R}$ is a valuation ring. Finally, if either (3.16) or (3.17) holds, we notice that the blowing up $\pi_{i+1}$ has strictly decreased the quantity $|s|+|t|$. Since this quantity cannot decrease indefinitely, after finitely many steps we will arrive either at (3.15) with $s$ and $t$ of the same sign or at (3.18), thus reducing the problem to one of the previous cases. Note also that if $f$ is of the form (3.15) with $s$ and $t$ of the same sign then the blowing up $\pi_{i+1}$ brings $f$ to the form (3.18). This completes the proof of (1).

(2) Conversely, let $R_{\mu}$ be a valuation ring such that $R_{i}<R_{\mu}$ for all $i \in \mathbb{N}$. Taking the direct limit as $i$ tends to infinity, we obtain $\bar{R}<R_{\mu}$. Now part (2) follows from (1) and the implication (a) $\Longrightarrow$ (c) of Remark 2.4 (2). However, we give below a direct proof of (2) for the sake of completeness.

If the sequence $\pi^{*}$ is finite, its last ring $R_{n}$ is a discrete valuation ring. Let $x_{n}$ be a local parameter of $R_{n}$. We have $\mu\left(x_{n}\right)>0$ since $\mu$ is centered at $\mathfrak{m}_{n}$. Now any element $f$ of $F^{*}$ can be written as $f=x_{n}^{s} u$ where $s \in \mathbb{Z}$ and $u$ is a unit of $R_{n}$ (hence also a unit of $R_{\mu}$ ) and therefore $f \in R_{n}$ if and only if $f \in R_{\mu}$.

If $\pi^{*}$ is infinite, let $f \in F^{*}$. As in the proof of part (1), there exists $i$ such that $f=x_{i}^{s} u$ or $f=y_{i}^{s} u$ with $x_{i}$ and $y_{i}$ local parameters in $R_{i}, s \in \mathbb{Z}$ and $u$ is a unit in $R_{i}$. Now since $\pi^{*}$ is infinite, we have $\nu\left(x_{i}\right)>0$ and $\nu\left(y_{i}\right)>0$ and $u$ is also a unit of $R_{\mu}$ since $R_{i}<R_{\mu}$. Hence to say that $f \in \bar{R}$ is equivalent to saying that $s \geq 0$, which is equivalent to saying that $\mu(f) \geq 0$. Therefore $\bar{R}=R_{\mu}$.

Corollary 3.11. The set of valuations of $F$ centered at $R$ is in a natural one-to-one correspondence with $\Pi(R)$. 
Proof. For the sake of completeness, we now give an explicit description of the element of $\Pi(R)$, associated to a given valuation $\mu$ by the above bijection and vice versa.

Let $\mu$ be a valuation centered at $R$. The center of $\mu$ in $R$ is $\xi_{0}:=\mathfrak{m}$. Consider the point blowing up $\pi_{1}^{*}: X_{1} \longrightarrow$ Spec $R$ along $\xi_{0}$. The center of $\mu$ in $X_{1}$ is the unique point $\xi_{1} \in X_{1}$ whose local ring $\mathcal{O}_{X_{1}, \xi_{1}}$ is dominated by $R_{\mu}$. Put $R_{1}:=\mathcal{O}_{X_{1}, \xi_{1}}$, and let $\mathfrak{m}_{1}:=\mathfrak{m}_{X_{1}, \xi_{1}}$ be its maximal ideal. If $\mathfrak{m}_{1}$ is principal, stop here. Otherwise, fix $x_{1}, y_{1} \in R_{1}$ such that $\mathfrak{m}_{1}=\left(x_{1}, y_{1}\right)$. We have $(R, \mathfrak{m})<\left(R_{1}, \mathfrak{m}_{1}\right)<\left(R_{\mu}, \mathfrak{m}_{\mu}\right)$. Now repeat the same procedure with $(R, \mathfrak{m})$ replaced by $\left(R_{1}, \mathfrak{m}_{1}\right)$. Continuing in this way we obtain the simple sequence $\pi^{*}(\mu)$ (finite or infinite) of local point blowings up of Spec $R$.

Now we have two cases:

Case 1: The ring $R$ does not contain an element $f$ satisfying condition (2) (b) in Lemma (3.8).

Letting $\bar{R}=\lim _{i \rightarrow \infty} R_{i}$, we have $\bar{R}=R_{\mu}$.

Conversely, take any element $\pi^{*} \in \Pi(R)$ and let $\bar{R}$ be as in Proposition 3.10, Let $\mu$ to be the valuation on $F$ with valuation $\operatorname{ring} \bar{R}$. It is clear that $\pi^{*}(\mu)$ described above is equal to $\pi^{*}$.

Case 2: The ring $R$ does contain an element $f$ satisfying condition (2) (b) in Lemma (3.8) then by Proposition (3.9) the valuation $\nu$ is uniquely determined by $\pi^{*}$.

Recall that $k$ denotes the residue field of $R$.

Theorem 3.12. There is a natural order preserving bijection between valuations of $F$ centered at $R$ and valuations of $k(x, y)$ centered at $k[x, y]_{(x, y)}$.

Proof. By Proposition 3.10, the set of valuations of $F$ centered at $R$ is in a natural one-to-one correspondence with $\Pi(R)$. Also by Proposition 3.10 applied to $k[x, y]_{(x, y)}$, the set of valuations of $k(x, y)$ centered at $k[x, y]_{(x, y)}$ is in a natural one-to-one correspondence with $\Pi\left(k[x, y]_{(x, y)}\right)$. Finally, there is a natural one-to-one correspondence between $\Pi(R)$ and $\Pi\left(k[x, y]_{(x, y)}\right)$. Clearly all those correspondences fit together to give a natural order preserving bijection between valuations of $F$ centered on $R$ and valuations of $k(x, y)$ centered on $k[x, y]_{(x, y)}$.

\section{A Complete Set of Key Polynomials}

Let $\tilde{\nu} \in \mathcal{V}$. Fix local coordinates $x$ and $y$ such that $\nu(x)=1$. Let $K=k(x)$. Let $\nu$ be the valuation of $k(x, y)$ corresponding to $\tilde{\nu}$ under the bijection of Theorem 3.12 .

The goal of this section is to construct a set of polynomials, complete for $\nu$ (the definition is given below). This set will be our main tool for constructing the valuative tree.

\subsection{Definition and Basic Properties of Key Polynomials}

For each strictly positive integer $b$, we write $\partial_{b}:=\frac{\partial^{b}}{b ! \partial y^{b}}$, the $b$-th formal derivative with respect to $y$. 
For each polynomial $P \in K[y]$, let $\epsilon_{\nu}(P):=\max _{b \in \mathbb{N}}\left\{\frac{\nu(P)-\nu\left(\partial_{b} P\right)}{b}\right\}$,

$$
I_{\nu}(P):=\left\{b \in \mathbb{N} \mid \frac{\nu(P)-\nu\left(\partial_{b} P\right)}{b}=\epsilon_{\nu}(P)\right\} .
$$

and $b_{\nu}(P):=\min I_{\nu}(P)$.

Definition 4.1. Let $Q$ be a monic polynomial in $K[y]$, with $\nu(Q) \geq \nu(y)$. We say that $Q$ is an abstract key polynomial for $\nu$ if for each polynomial $f$ satisfying

$$
\epsilon_{\nu}(f) \geq \epsilon_{\nu}(Q)
$$

we have $\operatorname{deg}(f) \geq \operatorname{deg}(Q)$.

For the rest of the paper, we will say key polynomial for abstract key polynomial.

For a monic polynomial $Q$ in $K[y]$ and a $g \in K[y]$ we can write $g$ in a unique way as

$$
g=\sum_{j=0}^{s} g_{j} Q^{j},
$$

with all the $g_{j} \in K[y]$ of degree strictly less than $\operatorname{deg}(Q)$.

Definition 4.2. For every monic polynomial $Q$ and every polynomial $g$ in $K[y]$, we call the expression (4.1) the $Q$-expansion of $g$. We define $\nu_{Q}(g):=\min _{0 \leq j \leq s} \nu\left(g_{j} Q^{j}\right)$. We call $\nu_{Q}$ the truncation of $\nu$ with respect to $Q$.

Proposition 4.3. (Proposition 12 of [3]) If $Q$ is a key polynomial for $\nu$ then $\nu_{Q}$ is a valuation.

The following proposition is a direct consequence of Proposition 19 [3] that states that each key polynomial for $\nu$ is $\nu$-irreducible.

Proposition 4.4. If $Q$ is a key polynomial for $\nu$ then $Q$ is irreducible.

Proposition 4.5. Every monic linear polynomial $Q$ in $K[y]$ is a key polynomial for $\nu$.

Proof. For any monic linear polynomial $Q \in K[y]$ and for any $c \in K$, we have

$$
\epsilon_{\nu}(Q)=\nu(Q)>-\infty=\epsilon_{\nu}(c)
$$

The first part of the next proposition is Theorem 27 of [3] and the second part is obvious.

Proposition 4.6. (1) Let $\mu$ be a valuation of $K(y)$ such that $\mu<\nu$, and let $Q$ be a monic polynomial of minimal degree in $y$ such that $\mu(Q)<\nu(Q)$. Then $Q$ is a key polynomial for $\nu$.

(2) Furthermore, we have $\mu<\nu_{Q} \leq \nu$ and the value group $\Gamma_{Q}$ of $\nu_{Q}$ is equal to $\Gamma_{\mu}+\beta \mathbb{Z}$ where $\Gamma_{\mu}$ is the value group of $\mu$ and $\beta=\nu(Q)$. 
Let $\mu$ be a valuation of $K(y)$ such that $\mu<\nu$, and let $Q$ be a monic polynomial of minimal degree in $y$ such that $\mu(Q)<\nu(Q)$. Let $\beta \in \overline{\mathbb{R}}$, with $\mu(Q)<\beta$.

We define a new valuation $\mu^{\prime}$ in the following way:

For a polynomial $g \in K[y]$, let $g=\sum_{j=0}^{s} g_{j} Q^{j}$ be the $Q$-expansion of $g$. Put

$$
\mu^{\prime}(g):=\min _{0 \leq j \leq s}\left\{\mu\left(g_{j}\right)+j \beta\right\} .
$$

We call $\mu^{\prime}$ the augmented valuation constructed from $\mu, Q$, and $\beta$, and we denote it by $[\mu, Q, \beta]$.

For further details on augmented valuations, see [17].

\subsection{A Complete Set of Key Polynomials: the Definition}

Let $\beta_{0}=\nu(x)=1$ and $\beta_{1}=\nu(y)$. Let $\Gamma_{\nu}=\nu(F) \subset \overline{\mathbb{R}}$ denote the augmented value group of $\nu$.

For an element $\beta \in \Gamma_{\nu}$, let $\mathbf{P}_{\beta}$ be as defined at thte end of $\S 2$, but with $F$ replaced by $K(y)$ :

$$
\mathbf{P}_{\beta}=\{f \in K(y) \mid \nu(f) \geq \beta\} .
$$

Definition 4.7. A complete set of key polynomials for $\nu$ is a set

$$
\mathbf{Q}=\left\{Q_{i}\right\}_{i \in I}
$$

where $I$ is a well ordered set, each $Q_{i}$ is a key polynomial in $K[y]$ for $\nu$, and for each $\beta \in \Gamma_{\nu}$ the additive group $\mathbf{P}_{\beta} \cap K[y]$ is generated by products of the form $a \prod_{j=1}^{s} Q_{i_{j}}^{\gamma_{j}}, a \in K$, such that $\sum_{j=1}^{s} \gamma_{j} \nu\left(Q_{i_{j}}\right)+\nu(a) \geq \beta$

In [6] it is proved that every valuation $\nu$ admits a complete set $\mathbf{Q}=\left\{Q_{i}\right\}_{i \in I}$ of key polynomials.

Remark 4.8. If $\mathbf{Q}=\left\{Q_{i}\right\}_{i \in I}$ is a complete set of key polynomials for $\nu$, we will always assume that the well ordering of $I$ has the following property: for $i<j$ in $I$, we have $\nu\left(Q_{i}\right)<\nu\left(Q_{j}\right)$.

\subsection{Basic Structure}

Let $\mu$ be a valuation of $K(y)$ with $\mu<\nu$. Suppose that the subset $\Gamma_{\mu+}$ of positive values of $\Gamma_{\mu}=\mu(K(y))$ is a well ordered set (with the standard order relation in $\overline{\mathbb{R}}$ ). Note that this assumption is equivalent to saying that $\Gamma_{\mu} \cong \mathbb{Z}$.

We will use the following notation:

1. Let $d_{\mu}(\nu)$ be the minimal degree of a monic polynomial $f$ in $K[y]$ satisfying $\mu(f)<\nu(f)$.

2. Put $\Phi_{\mu}(\nu):=\left\{Q \in K[y] \mid Q\right.$ is monic, $\left.\operatorname{deg}_{y}(Q)=d_{\mu}(\nu), \mu(Q)<\nu(Q)\right\}$.

3. Put $\Psi_{\mu}(\nu):=\nu\left(\Phi_{\mu}(\nu)\right)=\left\{\nu(Q) \in K[y] \mid Q\right.$ is monic, $\left.\operatorname{deg}_{y}(Q)=d_{\mu}(\nu), \mu(Q)<\nu(Q)\right\}$. 
Proposition 4.9. The set $\Psi_{\mu}(\nu)$ is contained in $\Gamma_{\mu+}$ or in $\Gamma_{\mu+} \cup\{\alpha\}$, where $\alpha$ is a maximal element of $\Psi_{\mu}(\nu)$ if it exists. Moreover, $\Psi_{\mu}(\nu)$ is bounded below by $d \mu(y) \geq 0$.

Proof. Let $d:=d_{\mu}(\nu)$. First we will show that $\Psi_{\mu}(\nu)$ is bounded below by $d \cdot \mu(y)$.

Let $\beta \in \Psi_{\mu}(\nu)$ and choose $Q \in \Phi_{\mu}(\nu)$ such that $\nu(Q)=\beta$.

Suppose that $\beta<d \mu(y)$ and write $Q=y^{d}+g$, with $g \in K[y], \operatorname{deg}_{y}(g)<d$.

Since $\mu\left(y^{d}\right)=d \mu(y)>\beta=\nu(Q)>\mu(Q)$, we have $\mu(Q)=\mu(g)$.

Since $\nu\left(y^{d}\right) \geq \mu\left(y^{d}\right)=d \mu(y)>\beta=\nu(Q)$, we have $\nu(Q)=\nu(g)$,

but $\nu(g)=\mu(g)$ by definition of $d$, therefore $\nu(Q)=\mu(Q)$, which is a contradiction. in $\Gamma_{\mu}$.

Now we will prove that any element $\beta \in \Psi_{\mu}(\nu)$ which is not a maximal element must be

Suppose that $\beta$ and $\alpha$ are two elements of $\Psi_{\mu}(\nu)$ such that $\beta<\alpha$.

Choose $Q$ and $Q^{\prime}$ in $\Phi_{\mu}(\nu)$ such that $\nu(Q)=\beta$ and $\nu\left(Q^{\prime}\right)=\alpha$.

Write $Q^{\prime}=Q+z$ with $z \in K[y], \operatorname{deg}_{y}(z)<d$.

Since $\alpha>\beta$, we have $\nu(Q)=\nu(z)$. But $\nu(z)=\mu(z)$ by definition of $d_{\mu}(\nu)$. Hence $\beta \in \Gamma_{\mu}$.

From Proposition 4.9 we see that $\Psi_{\mu}(\nu)$ is well ordered.

We will denote by $\beta_{\mu}(\nu)$ the smallest element of $\Psi_{\mu}(\nu)$.

Choose $Q \in \Phi_{\mu}(\nu)$ such that $\nu(Q)=\beta_{\mu}(\nu)$. By Proposition 4.6, $Q$ is a key polynomial for $\nu$, the truncation $\nu_{Q}$ is a valuation with $\mu<\nu_{Q} \leq \nu$, and the augmented value group $\Gamma_{Q}$ of $\nu_{Q}$ is $\Gamma_{\mu}+\beta_{\mu}(\nu) \mathbb{Z}$. Hence the set $\Gamma_{Q+}$ of positive values of $\Gamma_{Q}$ is a well ordered set.

If $\nu_{Q}<\nu$, we can repeat the same process as above with $\mu$ replaced by $\nu_{Q}$.

Moreover, the valuation $\nu_{Q}$ does not depend on the choice of $Q$, as we will prove in the following proposition.

Proposition 4.10. With the notation as above, if $Q^{\prime}$ is another polynomial in $\Phi_{\mu}(\nu)$ such that $\nu\left(Q^{\prime}\right)=\beta_{\mu}(\nu)$ then $\nu_{Q}=\nu_{Q^{\prime}}$.

Proof. Let $f$ be a polynomial of minimal degree such that $\nu_{Q}(f) \neq \nu_{Q^{\prime}}(f)$ and suppose that $\nu_{Q}(f)<\nu_{Q^{\prime}}(f)$.

Clearly $\operatorname{deg}_{y}(f) \geq d_{\mu}(\nu)$. Let $f=a_{s} Q^{s}+\cdots+a_{0}$ be the $Q$-expansion of $f$ and let

$$
g=a_{s-1} Q^{s-1}+\cdots+a_{0} .
$$

By definition of $\nu_{Q}$ we have $\nu\left(a_{i} Q^{i}\right) \geq \nu_{Q}(f)$ for each $0 \leq i \leq s$.

Suppose first that $\nu\left(a_{s} Q^{s}\right)>\nu_{Q}(f)$. Then $\nu_{Q}(f)=\nu_{Q}(g)$.

Since $\operatorname{deg}_{y}(g)<\operatorname{deg}_{y}(f)$, we have $\nu_{Q}(g)=\nu_{Q^{\prime}}(g)$. Therefore

$$
\nu_{Q^{\prime}}\left(a_{s} Q^{s}\right)=\nu\left(a_{s} Q^{s}\right)>\nu_{Q}(g)=\nu_{Q^{\prime}}(g) .
$$

This implies that $\nu_{Q^{\prime}}(f)=\nu_{Q^{\prime}}(g)$, that leads to $\nu_{Q^{\prime}}(f)=\nu_{Q}(f)$ which is a contradiction. We have proved that $\nu_{Q}(f)=\nu\left(a_{s} Q^{s}\right)$. 
Write $Q^{\prime}=Q+z$, with $\operatorname{deg}_{y}(z)<d_{\mu}(\nu)$. Then $f=a_{s}\left(Q^{\prime}-z\right)^{s}+\cdots+a_{0}$, and the $Q^{\prime}$-expansion of $f$ involves $a_{s} Q^{\prime s}$. Therefore we have $\nu\left(a_{s} Q^{\prime s}\right)=\nu_{Q^{\prime}}\left(a_{s} Q^{\prime s}\right) \geq \nu_{Q^{\prime}}(f)>\nu_{Q}(f)=\nu\left(a_{s} Q^{\prime s}\right)$, which is a contradiction.

Lemma 4.11. Let $f \in K[y]$ be such that

$$
\nu_{Q}(f)=\mu(f)
$$

and let $f=q Q+r$ be the Euclidean division of $f$ by $Q$ in $K[y]$. Then $\nu_{Q}(f)=\nu_{Q}(r)<\nu_{Q}(q Q)$. Proof. By definition of $\nu_{Q}$, we have

$$
\nu_{Q}(q Q) \geq \nu_{Q}(f)
$$

Suppose we have equality, aiming for contradiction:

$$
\nu_{Q}(q Q)=\nu_{Q}(f)
$$

Then

$$
\nu_{Q}(r) \geq \nu_{Q}(f)
$$

By definition of $\nu_{Q}$, we have

$$
\mu(r)=\nu_{Q}(r) .
$$

Combining (4.2), (4.5) and (4.6), we obtain $\mu(f) \leq \mu(r)$. Then

$$
\mu(f) \leq \mu(q Q) .
$$

We have

$$
\nu_{Q}(q Q)>\mu(q Q) .
$$

Combining (4.2), (4.4), (4.7) and (4.8), we get $\nu_{Q}(q Q)>\mu(q Q) \geq \mu(f)=\nu_{Q}(f)=\nu_{Q}(q Q)$, which is a contradiction.

Proposition 4.12. (Theorem 9.4 [10], Theorem 1.11 [17]) Let $Q^{\prime}$ be a monic polynomial of minimal degree among those satisfying

$$
\nu_{Q}\left(Q^{\prime}\right)<\nu\left(Q^{\prime}\right)
$$

Then the $Q$-expansion of $Q^{\prime}$ is given by $Q^{\prime}=Q^{s}+a_{s-1} Q^{s-1} \cdots+a_{0}$ with

$$
\nu_{Q}\left(Q^{\prime}\right)=s \beta_{\mu}(\nu)=\nu\left(a_{0}\right) .
$$

Proof. First, let $Q^{\prime}=q Q+a_{0}$ be the Euclidean division of $Q^{\prime}$ by $Q$. We have

$$
\nu\left(Q^{\prime}\right)>\nu_{Q}\left(Q^{\prime}\right)=\inf \left\{\nu_{Q}(q Q), \nu\left(a_{0}\right)\right\}
$$

by definition of $\nu_{Q}$. Hence $\nu(q Q)=\nu\left(a_{0}\right)=\nu_{Q}\left(Q^{\prime}\right)$.

Since $\operatorname{deg}_{y}\left(a_{s}\right)<d_{\mu}(\nu)$ and $Q$ is irreducible in $K[y]$ by Proposition 4.4, there exist $g$ and $h$ in $K[y]$ with $\operatorname{deg}_{y}(g)<d_{\mu}(\nu)$ and $g a_{s}+h Q=1$. Now $g a_{s}=-h Q+1, \nu_{Q}\left(a_{s}\right)=\mu\left(a_{s}\right)$ and $\nu_{Q}(g)=\mu(g)$, therefore by Lemma 4.11 we have $\nu\left(g a_{s}\right)=\nu(1)<\nu(-h Q)$. 
Now for each $j, 0 \leq j \leq s-1$, let $g a_{j}=q_{j} Q+r_{j}$ be the Euclidean division of $g a_{j}$ by $Q$ in $K[y]$. Since $\nu_{Q}\left(a_{j}\right)=\mu\left(a_{j}\right)$ and $\nu_{Q}(g)=\mu(g)$, by Lemma4.11 we have $\nu\left(g a_{j}\right)=\nu\left(r_{j}\right)<\nu\left(q_{j} Q\right)$.

Consider the polynomial $Q^{\prime \prime}=Q^{s}+r_{s-1} Q^{s-1}+\cdots+r_{0}$.

We have $Q^{\prime \prime}-g Q^{\prime}=\left(r_{s}-g a_{s}\right) Q^{s}+\left(r_{s-1}-g a_{s-1}\right) Q^{s-1}+\cdots+r_{0}-g a_{0}$, with $r_{s}=1$.

Therefore

$$
\nu_{Q}\left(Q^{\prime \prime}-g Q^{\prime}\right) \geq \inf _{0 \leq j \leq s}\left\{\nu\left(r_{j}-g a_{j}\right)+j \beta_{\mu}(\nu)\right\}>\inf _{0 \leq j \leq s}\left\{\nu\left(r_{j}\right)+j \beta_{\mu}(\nu)\right\}=\nu_{Q}\left(Q^{\prime \prime}\right)
$$

and $\nu_{Q}\left(Q^{\prime \prime}-g Q^{\prime}\right)>\nu_{Q}\left(Q^{\prime \prime}\right)=\nu_{Q}\left(g Q^{\prime}\right)$.

If $\nu\left(Q^{\prime \prime}\right)=\nu_{Q}\left(Q^{\prime \prime}\right)$ then $\nu\left(Q^{\prime \prime}-g Q^{\prime}\right) \geq \nu_{Q}\left(Q^{\prime \prime}-g Q^{\prime}\right)>\nu_{Q}\left(Q^{\prime \prime}\right)=\nu\left(Q^{\prime \prime}\right)$ and we have $\nu\left(Q^{\prime \prime}\right)=\nu\left(g Q^{\prime}\right)>\nu_{Q}\left(g Q^{\prime}\right)=\nu_{Q}\left(Q^{\prime \prime}\right)$, which is impossible. Hence $\nu\left(Q^{\prime \prime}\right)>\nu_{Q}\left(Q^{\prime \prime}\right)$.

Since $Q^{\prime}$ is chosen of minimal degree we must have $\operatorname{deg}_{y}\left(Q^{\prime \prime}\right) \geq \operatorname{deg}_{y}\left(Q^{\prime}\right)$, but this implies that $\operatorname{deg}_{y} a_{s}=0$ and $a_{s}=1$.

We still have to prove that $\nu_{Q}\left(Q^{\prime}\right)=\nu\left(Q^{s}\right)$. By definition of $\nu_{Q}$ we have $\nu\left(Q^{s}\right) \geq \nu_{Q}\left(Q^{\prime}\right)$.

Suppose that $\nu\left(Q^{s}\right)>\nu_{Q}\left(Q^{\prime}\right)$, then $\nu_{Q}\left(Q^{\prime}\right)=\nu_{Q}(f)$, where $f=Q^{\prime}-Q^{s}$.

But since $\operatorname{deg}_{y}(f)<\operatorname{deg}_{y}\left(Q^{\prime}\right)$, we have $\nu_{Q}(f)=\nu(f)$. We obtain

$$
\nu\left(Q^{s}\right)=\nu_{Q}\left(Q^{s}\right)>\nu_{Q}\left(Q^{\prime}\right)=\nu_{Q}(f)=\nu(f) .
$$

This implies that $\nu\left(Q^{\prime}\right)=\nu(f)$, which leads to $\nu\left(Q^{\prime}\right)=\nu_{Q}\left(Q^{\prime}\right)$, a contradiction.

Corollary 4.13. If $Q^{\prime} \in K[y]$ is monic with $\operatorname{deg}_{y}\left(Q^{\prime}\right)=d_{\mu}(\nu)$ and $\nu\left(Q^{\prime}\right)>\nu_{Q}\left(Q^{\prime}\right)$ then $\nu_{Q}\left(Q^{\prime}\right)=\beta_{\mu}(\nu)$.

Proof. This is a special case of Proposition 4.12 when $\operatorname{deg}_{y}\left(Q^{\prime}\right)=d_{\mu}(\nu)$.

Proposition 4.14. Let $\left\{Q_{i}\right\}_{i \in I}$ be a set of key polynomials for $\nu$, with $I$ a well ordered set and $\operatorname{deg}_{y}\left(Q_{i}\right) \leq \operatorname{deg}_{y}\left(Q_{j}\right)$ for $i<j$ in I. Let $\nu_{i}:=\nu_{Q_{i}}$ be the truncation associated with each $Q_{i}$. Then the set $\left\{Q_{i}\right\}_{i \in I}$ is a complete set of key polynomials if and only if for each polynomial $f \in K[y]$ there exists an element $i \in I(\nu)$ such that $\nu(f)=\nu_{i}(f)$ and $\operatorname{deg}_{y} Q_{i} \leq \operatorname{deg}_{y} f$.

Proof. To say that $\left\{Q_{i}\right\}_{i \in I(\nu)}$ is a complete set of key polynomials is equivalent to saying that every $f \in K[x]$ can be written in the form

$$
f=\sum_{\gamma} a_{\gamma} \prod_{j=1}^{s} Q_{j}^{\gamma_{j}}
$$

where $s$ is a strictly positive integer, $\gamma=\left(\gamma_{1}, \ldots, \gamma_{s}\right)$ ranges over a finite subset of $\mathbb{N}^{s}, a_{\gamma} \in K$ and

$$
\sum_{j=1}^{s} \gamma_{j} \nu\left(Q_{j}\right)+\nu(a) \geq \nu(f) .
$$

Proof of "if". Assume that for each $f \in K[x]$ there exists an integer $i$ as in the Proposition. We will construct the expression (4.9) recursively in $\operatorname{deg}_{y} f$. Assume that an expression of the form 
(4.9) exists for every polynomial of degree strictly less than $\operatorname{deg}_{y} f$. Put $\beta=\nu(f)$ and let $i$ be such that $\beta=\nu_{i}(f)$ and $\operatorname{deg}_{y} Q_{i} \leq \operatorname{deg}_{y} f$. Write

$$
f=\sum_{j=0}^{s_{i}} c_{j} Q_{i}^{j},
$$

where each $c_{j} Q_{i}^{j} \in P_{\beta}$ and $\operatorname{deg}_{y} c_{j}<\operatorname{deg}_{y} Q_{i} \leq \operatorname{deg}_{y} f$. By the induction assumption, each of the $c_{j}$ admits an expansion of the form (4.9). Substituting all of these expansions into (4.10), we obtain the desired expansion (4.9) of $f$. This completes the proof of "if".

Proof of "only if". Conversely, take $f \in K[y]$. Let $\beta=\nu(f)$. Write $f$ in the form (4.9). Then

$$
\beta=\nu(f) \geq \nu_{s}(f) \geq \min \left\{\nu_{s}\left(c_{\gamma} \prod Q_{j}^{\gamma_{j}}\right)\right\}=\min \left\{\nu\left(c_{\gamma} \prod Q_{j}^{\gamma_{j}}\right)\right\} \geq \beta .
$$

Thus all the inequalities in the above formula are equalities, so the natural number $i:=s$ satisfies the conclusion of "only if".

\subsection{Construction of a Complete Set of Key Polynomials}

First we put $Q_{1}:=y$ and $d_{1}(\nu)=1$. By Proposition 4.5, $Q_{1}$ is a key polynomial for $\nu$. Consider the valuation $\nu_{1}:=\nu_{Q_{1}}$. We have $\nu_{1} \leq \nu$. If $\nu_{1}=\nu$ then the algorithm stops here, we put $I(\nu)=\{1\}$ and $\left\{Q_{i}\right\}_{i \in I(\nu)}=\left\{Q_{1}\right\}$. We will prove in Proposition 4.16 below that $\left\{Q_{i}\right\}_{i \in I(\nu)}$ is complete for $\nu$.

Now suppose that $\nu_{1}<\nu$. Then we can apply the results of 4.3 to $\mu=\nu_{1}$. Put $d_{2}(\nu)=d_{\nu_{1}}(\nu), \Phi_{1}(\nu)=\Phi_{\nu_{1}}(\nu), \Psi_{1}(\nu)=\Psi_{\nu_{1}}(\nu)$ and $\beta_{2}(\nu)=\beta_{\nu_{1}}(\nu)$.

Choose $Q_{2} \in \Phi_{1}(\nu)$ such that $\nu\left(Q_{2}\right)=\beta_{2}(\nu)$ and let $\nu_{2}:=\nu_{Q_{2}}$.

We have $\nu_{1}<\nu_{2} \leq \nu$. If $\nu_{2}=\nu$, then the algorithm stops here, we put $I(\nu)=\{1,2\}$ and $\left\{Q_{i}\right\}_{i \in I(\nu)}=\left\{Q_{1}, Q_{2}\right\}$. By Proposition 4.16 below, $\left\{Q_{i}\right\}_{i \in I(\nu)}$ is complete for $\nu$.

Otherwise, if $\nu_{2}<\nu$, we can apply the results of 4.3 to $\mu=\nu_{1}$ and repeat the same process with $\nu_{1}$ replaced by $\nu_{2}$.

Assume that for a certain natural number $n \geq 2$ a set $\left\{Q_{i}\right\}_{i \geq n}$ has been constructed. If $\nu_{n}=\nu$ then, by Proposition 4.14 and Proposition 4.16 below, $\left\{Q_{i}\right\}_{i \geq n}$ is complete for $\nu$. The construction stops here.

Otherwise, we have $\nu_{n}<\nu$. Let us apply the results of $\$ 4.3$ to $\mu=\nu_{n}$. Put

$$
d_{n+1}(\nu)=d_{\nu_{n}}(\nu),
$$

$\Phi_{n}(\nu)=\Phi_{\nu_{n}}(\nu), \Psi_{n}(\nu)=\Psi_{\nu_{n}}(\nu)$ and $\beta_{n+1}(\nu)=\beta_{\nu_{n}}(\nu)$.

Choose $Q_{n+1} \in \Phi_{n}(\nu)$ such that $\nu\left(Q_{n+1}\right)=\beta_{n+1}(\nu)$ and let $\nu_{n+1}:=\nu_{Q_{n+1}}$.

Repeating this process, there are two possibilities. The first is that we find valuations $\left\{\nu_{i}\right\}_{i \leq n}$ and key polynomials $\left\{Q_{i}\right\}_{i \leq n}$ such that $\nu_{n}=\nu$. The second is that we construct an infinite set $\left\{Q_{i}\right\}_{i \in \mathbb{N}}$ of key polynomials and valuations $\left\{\nu_{i}\right\}_{i \in \mathbb{N}}$. We will study this case after Proposition 4.16. 
Remark 4.15. Let $\left\{Q_{i}\right\}_{i \in I(\nu)}$ be the set constructed above, with $I(\nu)=\{1,2, \ldots\}$ (possibly infinite). Even though the polynomials $\left\{Q_{i}\right\}_{i \in I(\nu)}$ are not uniquely determined, their degrees $\left\{d_{i}(\nu)\right\}_{i \in I(\nu)}$, their values $\left\{\beta_{i}(\nu)\right\}_{i \in I(\nu)}$, the associated valuations $\left\{\nu_{i}\right\}_{i \in I(\nu)}$ are uniquely determined by $\nu$, from the construction above and by Proposition 4.10. As well, the sets $\Phi_{i}(\nu)$ and $\Psi_{i}(\nu)$, for $i \in I(\nu)$, are uniquely determined by $\nu$ by construction.

Proposition 4.16. Let $\left\{Q_{i}\right\}_{i \in I(\nu)}$ be the set constructed above, with $I(\nu)$ possibly infinite. Let $f \in K[y]$ and suppose there exists $i_{0} \in I(\nu)$ such that $\operatorname{deg}_{y}(f)<\operatorname{deg}_{y}\left(Q_{i_{0}}\right)$. There exists $i<i_{0}$ such that $\operatorname{deg}_{y}\left(Q_{i}\right) \leq \operatorname{deg}_{y}(f)$ and $\nu_{i}(f)=\nu(f)$.

Proof. Multiplying $f$ by a non-zero element of $K$ does not change the problem, therefore we may assume that $f$ is monic.

Now let $i$ be such that $\left(d_{i}(\nu), \beta_{i}(\nu)\right)=\max \left\{j \in I(\nu) \mid\left(d_{j}(\nu), \beta_{j}(\nu)\right) \leq_{l e x}\left(\operatorname{deg}_{y}(f), \nu(f)\right)\right\}$. Now by construction, if $i+1$ does not exist, then $\nu(f)=\nu_{i}(f)$. Otherwise, if $i+1 \in I(\nu)$, then $\left(d_{i+1}(\nu), \beta_{i+1}(\nu)\right)>\left(\operatorname{deg}_{y}(f), \nu(f)\right)$, and by definition of $Q_{i+1}$ we have $\nu(f)=\nu_{i}(f)$.

Suppose that $\left\{Q_{i}\right\}_{i \in I(\nu)}$ is constructed as above with $I(\nu)=\mathbb{N}$. Two cases are possible.

Case 1: The set $\Phi_{i}(\nu)$ is finite for each $i \in I(\nu)$.

Case 2: There exists $i \in I(\nu)$ such that $\Phi_{i}(\nu)$ is infinite.

Proposition 4.17. If $\beta_{i+1}(\nu)$ is a maximal element of $\Psi_{i}(\nu)$ then $\Psi_{i}(\nu)=\left\{\beta_{i+1}(\nu)\right\}$ and

$$
\Psi_{i}(\nu) \cap \Psi_{i+1}(\nu)=\emptyset .
$$

Moreover, if $\Psi_{i+1}(\nu) \neq \emptyset$ then $d_{i+2}(\nu)>d_{i+1}(\nu)$ and $\beta_{i+2}(\nu)>\beta_{i+1}(\nu)$. If $\beta_{i+1}(\nu)$ is not maximal in $\Psi_{i}(\nu)$, then $\Psi_{i+1}(\nu)=\Psi_{i}(\nu) \backslash\left\{\beta_{i+1}(\nu)\right\}$ and $d_{i+2}(\nu)=d_{i+1}(\nu)$.

Proof. First suppose that $\beta_{i+1}(\nu)$ is maximal in $\Psi_{i}(\nu)$. Since by definition $\beta_{i+1}(\nu)$ is the minimal element of $\Psi_{i}(\nu)$ and $\Psi_{i}(\nu)$ is totally ordered, we have $\Psi_{i}(\nu)=\left\{\beta_{i+1}\right\}$.

Now if $\Psi_{i+1}(\nu)=\emptyset$, the equality (4.11) hods trivially. Thus we will assume that

$$
\Psi_{i+1}(\nu) \neq \emptyset .
$$

We will first prove that $d_{i+2}(\nu)>d_{i+1}(\nu)$.

By construction, we have $d_{i+2}(\nu) \geq d_{i+1}(\nu)$. Aiming for contradiction, suppose that we have equality. Take an element $Q \in \Phi_{i+1}(\nu)$.

We have $\operatorname{deg}_{y}(Q)=d_{i+2}(\nu)=d_{i+1}(\nu)$ and $\nu(Q)>\nu_{i+1}(Q) \geq \nu_{i}(Q)$, therefore $Q \in \Phi_{i}(\nu)$, by definition of $\Phi_{i}(\nu)$. Hence $\nu(Q) \in \Psi_{i}(\nu)=\left\{\beta_{i+1}(\nu)\right\}$.

On the other hand, $Q$ satifies the hypothses on $Q^{\prime}$ in Corollary 4.13, Therefore

$$
\nu_{i+1}(Q)=\beta_{i+1}(\nu) .
$$

This implies that $\nu(Q)=\nu_{i+1}(Q)$ which contradicts the fact that $Q \in \Phi_{i+1}(\nu)$.

We have proved that $d_{i+1}(\nu)<d_{i+2}(\nu)$. Now the fact that $\beta_{i+2}(\nu)>\beta_{i+1}(\nu)$ follows from Proposition 4.12, since $\beta_{i+2}(\nu)>\nu_{i+1}\left(Q_{i+2}\right)=s \beta_{i+1}$, where $s=\operatorname{deg}_{Q_{i}} Q_{i+1}$. 
Since $\beta_{i+2}(\nu)>\beta_{i+1}(\nu)$, we have $\Psi_{i}(\nu) \cap \Psi_{i+1}(\nu)=\emptyset$.

Next, suppose that $\beta_{i+1}(\nu)$ is not a maximal element in $\Psi_{i}(\nu)$. Let $\beta \in \Psi_{i}(\nu) \backslash\left\{\beta_{i+1}(\nu)\right\}$. Choose $Q \in \Phi_{i}(\nu)$ such that $\nu(Q)=\beta$ and write $Q=Q_{i+1}+z$. Since $\beta>\beta_{i+1}(\nu)$, we have $\nu(z)=\beta_{i+1}(\nu)$ and $\nu_{i+1}(Q)=\beta_{i+1}(\nu)<\beta=\nu(Q)$. We have proved that $Q \in \Phi_{i+1}(\nu)$, therefore $d_{i+2}(\nu)=d_{i+1}(\nu)$ and $\beta \in \Psi_{i+1}(\nu)$. Thus $\Psi_{i}(\nu) \backslash\left\{\beta_{i+1}(\nu)\right\} \subset \Psi_{i+1}(\nu)$.

Now let $\beta \in \Psi_{i+1}(\nu)$. By Corollary 4.13, we have $\beta>\beta_{i+1}(\nu)$. Take an element

$$
Q \in \Phi_{i+1}(\nu)
$$

such that $\nu(Q)=\beta$. We have $\operatorname{deg}_{y}(Q)=d_{i+2}(\nu)=d_{i+1}(\nu)$, and $\nu(Q)>\nu_{i+1}(Q) \geq \nu_{i}(Q)$, hence $Q \in \Phi_{i}(\nu)$ and $\beta \in \Psi_{i}(\nu) \backslash\left\{\beta_{i+1}(\nu)\right\}$.

Corollary 4.18. If $\Psi_{i}(\nu)$ is infinite for some $i \in I(\nu)$, then $\Psi_{i+1}(\nu)$ is infinite and

$$
\Psi_{i+1}(\nu) \subset \Psi_{i}(\nu) .
$$

Corollary 4.19. Let $i_{0}=\inf \left\{i \in \mathbb{N} \mid \# \Psi_{i}(\nu)=\infty\right\}$, then for each $i \geq i_{0}$, the value group $\Gamma_{i}$ of $\nu_{i}$ is equal to the value group $\Gamma_{i_{0}}$ of $\nu_{i_{0}}$.

Proof. This is a direct consequence of Proposition 4.9 (2) and Corollary 4.18

First, suppose we are in Case 1:

Proposition 4.20. The degrees $d_{i}(\nu)$ are unbounded in $\mathbb{N}$.

Proof. Take an element $i \in I(\nu)$. We will prove that there exists $j \in I(\nu)$ such that

$$
d_{j}(\nu)>d_{i+1}(\nu)
$$

Since $\Psi_{i}(\nu)$ is finite, it admits a maximal element $\alpha$. If $\beta_{i+1}(\nu)=\alpha$ then, by Proposition 4.17 , we have $d_{i+2}(\nu)>d_{i+1}(\nu)$. Suppose that $\beta_{i+1}(\nu)$ is not maximal. By Proposition 4.17 we have $\Psi_{i+1}(\nu)=\Psi_{i}(\nu) \backslash\left\{\beta_{i+1}(\nu)\right\}$, therefore $\alpha$ is also the maximal element of $\Psi_{i+1}(\nu)$. Now repeat the same reasoning: if $\beta_{i+2}(\nu)=\alpha$, then $d_{i+3}(\nu)>d_{i+2}(\nu)=d_{i+1}(\nu)$, otherwise we have $\Psi_{i+2}(\nu)=\Psi_{i+1}(\nu) \backslash\left\{\beta_{i+2}(\nu)\right\}$ and $\alpha$ is the maximal element of $\Psi_{i+2}(\nu)$. The process must end since $\Psi_{i}(\nu)$ is finite.

Theorem 4.21. The set of key polynomials $\left\{Q_{i}\right\}_{i \in I(\nu)}$ is complete for $\nu$.

Proof. Let $f \in K[y]$. By Proposition 4.20, there exists $i \in I(\nu)$ such that $d_{i}(\nu)>\operatorname{deg}_{y}(f)$. Now the result follows from Proposition 4.14 and Proposition 4.16 .

Suppose we are in Case 2.

Theorem 4.22. $\quad$ 1. If $\nu^{-1}(\infty)=\{0\}$ then the set $\left\{Q_{i}\right\}_{i \in I(\nu)}$ is complete for $\nu$.

2. If $\nu^{-1}(\infty) \neq\{0\}$ then there exists a key polynomial $Q_{\omega}$ for $\nu$, that generates the ideal $\nu^{-1}(\infty)$ and it is of minimal degree such that $\nu_{i}\left(Q_{\omega}\right)<\nu\left(Q_{\omega}\right)$ for all $i \in \mathbb{N}$. Moreover, the set $\left\{Q_{i}\right\}_{i \in I(\nu)} \cup\left\{Q_{\omega}\right\}$ is complete for $\nu$. 
Proof. Fix a polynomial $f \in K[y]$ such that $\nu(f)<\infty$. We have the following inequalities:

$$
\nu_{1}(f) \leq \cdots \leq \nu_{i}(f) \leq \cdots \leq \nu(f)<\infty .
$$

By Corollary 4.19, all those values belongs to the value group $\Gamma_{i_{0}}$ of $\nu_{i_{0}}$, where $i_{0} \in I(\nu)$ is defined in Corollary 4.19, Now $\Gamma_{i_{0}}=\beta_{0} \mathbb{Z}+\beta_{1} \mathbb{Z}+\cdots+\beta_{i_{0}} \mathbb{Z}$ is discrete, hence there exists a certain integer $j$ such that $\nu_{i}(f)=\nu(f)$ for any $i \geq j \in \mathbb{N}$.

Now 1) follows from Proposition 4.14.

To prove 2), suppose that $\nu^{-1}(\infty) \neq\{0\}$. The set $\nu^{-1}(\infty)$ is an ideal in $K[y]$, it can be generated by one element. Choose $Q_{\omega}$ to be a monic polynomial that generates $\nu^{-1}(\infty)$. The polynomial $Q_{\omega}$ has minimal degree among the polynomials in $\nu^{-1}(\infty)$.

We have $\epsilon_{\nu}\left(Q_{\omega}\right)=\infty$, and $Q_{\omega}$ of minimal degree with this property, hence $Q_{\omega}$ is a key polynomial for $\nu$.

We have $\nu=\nu_{\omega}:=\nu_{Q_{\omega}}$, and for any polynomial $f \in K[y]$, if $f \notin \nu^{-1}(\infty)$, by the discussion at the beginning of the proof, there exists $i \in I(\nu)$ such that $\nu_{i}(f)=\nu(f)$, otherwise, if $f \in \nu^{-1}(\infty)$, then $\nu(f)=\nu_{\omega}(f)=\infty$. Hence the set $\left\{Q_{i}\right\}_{i \in \mathbb{N}} \cup\left\{Q_{\omega}\right\}$ is complete for $\nu$ Proposition 4.14 .

For the rest of the paper if $Q_{\omega}$ exists, we put $I(\nu)=\mathbb{N} \cup\{\omega\}$.

We denote:

1. $D(\nu):=\max _{i \in I(\nu)}\left\{d_{i}(\nu)\right\}$, if this maximum exists; otherwise, we put $D(\nu)=\infty$.

2. $N(\nu)$ the maximal element of $I(\nu)$, if this maximum exists, otherwise, we put $N(\nu)=\infty$.

Remark 4.23. From the construction above we see that:

1. $N(\nu)=\infty$ if and only if $I(\nu)=\mathbb{N}$.

2. If $D(\nu)=\infty$ then $I(\nu)=\mathbb{N}$.

3. If $D(\nu)<\infty$ and $(N(\nu)=\infty$ or $N(\nu)=\omega)$, we are in the case where there exists $i \in I(\nu)$ such that $\# \Psi_{i}(\nu)=\infty$.

\section{The order relation on $\mathcal{V}$}

\subsection{Invariants of comparable valuations}

Let $\tilde{\mu}$ and $\tilde{\nu}$ be two elements of $\mathcal{V}$ with $\tilde{\mu}<\tilde{\nu}$. Choose local coordinates $x$ and $y$ such that $\tilde{\nu}(x)=\tilde{\mu}(x)=1$.

Put $K=k(x)$ and let $\mu$ and $\nu$ be the valuations of $K(y)$, corresponding to $\tilde{\mu}$ and $\tilde{\nu}$, respectively.

Let $\left\{Q_{i}\right\}_{i \in I(\nu)}$ be a complete set of key polynomials associated to $\nu$. 
Lemma 5.1. There exists $i \in I(\nu)$ such that $\mu\left(Q_{i}\right)<\nu\left(Q_{i}\right)$.

Proof. Suppose that for all $i \in I(\nu)$ we have $\mu\left(Q_{i}\right)=\nu\left(Q_{i}\right)$.

Since $\nu>\mu$, there exists $f \in K[y]$ such that $\nu(f)>\mu(f)$. Choose $f \in K[y]$ of minimal degree among the polynomials having this property.

Since $\left\{Q_{i}\right\}_{i \in I(\nu)}$ is complete for $\nu$, there exists $i \in I(\nu)$ such that $\nu(f)=\nu_{i}(f)$.

Let $f=q Q_{i}+r$ be the Euclidean division of $f$ by $Q_{i}$.

We have $\nu(f)>\mu(f) \geq \inf \left\{\mu\left(q Q_{i}\right), \mu(r)\right\}=\inf \left\{\nu\left(q Q_{i}\right), \nu(r)\right\} \geq \nu_{i}(f)$, which is a contradiction.

Let $i_{0}:=\min \left\{i \in I(\nu) \mid \mu\left(Q_{i}\right)<\nu\left(Q_{i}\right)\right\}$.

Proposition 5.2. If $i_{0}=1$, then $I(\mu)=\{1\}$ and $\mu=\mu_{1}<\nu_{1}$.

Proof. Since $i_{0}=1$, we have $\mu(y)<\nu(y)$. It is sufficient to prove that $\mu=\mu_{1}$.

Suppose there exists $f \in K[y]$, with $\mu(f)>\mu_{1}(f)$. Choose $f$ of minimal degree satisfying $\mu(f)>\mu_{1}(f)$ and let $f=q y+r$ be the Euclidean division of $f$ by $y$.

We have $\mu(f)>\mu_{1}(f)=\inf \{\mu(q y), \mu(r)\}$, therefore, $\mu(f)>\mu(q y)=\mu(r)$. Since $\nu(f) \geq \mu(f)$, we have $\nu(f)>\mu(r)=\nu(r)$. This implies that $\nu(f)>\nu(q y)=\nu(r)$. Finally, we get $\nu(q y)=\nu(r)=\mu(r)=\mu(q y)$. But $\nu(q y)=\nu(q)+\nu(y)>\mu(q)+\mu(y)=\mu(q y)$, and we have a contradiction.

Proposition 5.3. If $i_{0}>1$ then for any $i \in I(\nu)$ with $i<i_{0}$, we have $i \in I(\mu), \mu_{i}=\mu_{Q_{i}}=\nu_{i}$, $\beta_{i}(\mu)=\beta_{i}(\nu)$ and $d_{i}(\mu)=d_{i}(\nu)$.

Proof. Since $i_{0}>1$, we have $\mu(y)=\nu(y)$, hence $\mu_{1}=\nu_{1}, \beta_{1}(\mu)=\beta_{1}(\nu)$ and $d_{1}(\mu)=d_{1}(\nu)$.

Take an integer $i, 1<i<i_{0}$ (in particular $i \in \mathbb{N}$ ), and suppose inductively that for all $j$, $1 \leq j<i$, we have $\nu_{j}=\mu_{j}, \beta_{j}(\mu)=\beta_{j}(\nu)$ and $d_{j}(\mu)=d_{j}(\nu)$.

We will first prove that $d_{i}(\mu)=d_{i}(\nu)$.

If $f$ is a monic polynomial with $\mu(f)>\mu_{i-1}(f)=\nu_{i-1}(f)$, then $\nu(f) \geq \mu(f)>\nu_{i-1}(f)$, hence $d_{i}(\nu) \leq d_{i}(\mu)$.

To prove the equality, we will prove that $\mu\left(Q_{i}\right)>\mu_{i-1}\left(Q_{i}\right)$. Indeed, by definition of $i_{0}$ we have $\mu\left(Q_{i}\right)=\nu\left(Q_{i}\right)$ since $i<i_{0}$.

Hence $\mu\left(Q_{i}\right)=\nu\left(Q_{i}\right)>\nu_{i-1}\left(Q_{i}\right)=\mu_{i-1}\left(Q_{i}\right)$.

Therefore $d_{i}(\nu)=d_{i}(\mu)$.

Now to prove that $\beta_{i}(\mu)=\beta_{i}(\nu)$, we still have to prove that if $f$ is a monic polynomial with $\mu(f)>\mu_{i-1}(f)$ and $\operatorname{deg}_{y}(f)=d_{i}(\mu)$, then $\mu(f) \geq \mu\left(Q_{i}\right)$. In this case, we will have $\beta_{i}(\mu)=\mu\left(Q_{i}\right)$, and since by definition of $i_{0}, \mu(Q)=\nu(Q)$, we get the desired equality.

Let $f$ be such a polynomial. Write $f=Q_{i}+g$ with $\operatorname{deg}_{y}(g)<d_{i}(\mu)$.

If $\mu(f)<\mu\left(Q_{i}\right)$ then $\mu(f)=\mu(g)=\mu_{i-1}(g)=\nu_{i-1}(g)=\nu(g)$ and

$$
\nu\left(Q_{i}\right) \geq \mu\left(Q_{i}\right)>\mu(f)=\nu(g) .
$$


Hence $\nu(g)=\nu(f)$ and $\nu\left(Q_{i}\right)>\nu(f)$.

We have proved that $\beta_{i}(\nu)>\nu(f)$ and $\nu(f) \geq \mu(f)>\mu_{i-1}(f)=\nu_{i-1}(f)$, which contradicts the definition of $\beta_{i}(\nu)$.

Since $Q_{i}$ is monic of degree $d_{i}(\mu)$ with $\mu\left(Q_{i}\right)=\beta_{i}(\mu)$, we have $\mu_{i}=\mu_{Q_{i}}$. Since $\beta_{i}(\mu)=$ $\beta_{i}(\nu)$ then $\mu_{i}=\nu_{i}$.

Proposition 5.4. We have $i_{0}<\omega$. In other words, $i_{0} \in \mathbb{N}$.

Proof. Suppose that $\omega \in I(\nu)$ and $i_{0}=\omega$.

By Remark 4.23 (3) and Corollary 4.19, there exists $i_{1} \in \mathbb{N}$ such that $\beta_{i}(\nu) \in \Gamma_{i_{1}}$ for all $i \geq i_{1}$, with $\Gamma_{i_{1}}=\mathbb{Z}+\beta_{1} \mathbb{Z}+\cdots+\beta_{i_{1}} \mathbb{Z} \subset \mathbb{R}$. Hence for all $i \in \mathbb{N}$, we have $\nu_{i}\left(Q_{\omega}\right) \in \Gamma_{i_{1}}$.

Let us show that

$$
\nu_{1}\left(Q_{\omega}\right)<\nu_{2}\left(Q_{\omega}\right)<\cdots<\nu\left(Q_{\omega}\right)=\infty .
$$

Indeed, assume that there exists $i \in I(\nu) \backslash\{\omega\}$, such that $\nu_{i}\left(Q_{\omega}\right)=\nu_{i+1}\left(Q_{\omega}\right)$, aiming for contradiction. Write $Q_{\omega}=q Q_{i+1}+r$ the Euclidean Division of $Q_{\omega}$ by $Q_{i+1}$. We have $\nu_{i+1}\left(q Q_{i+1}\right)>\nu_{i}\left(q Q_{i+1}\right) \geq \inf \left\{\nu_{i}\left(Q_{\omega}\right), \nu_{i}(r)\right\}=\inf \left\{\nu_{i+1}\left(Q_{\omega}\right), \nu_{i+1}(r)\right\}$, hence

$$
\nu_{i+1}\left(q Q_{i+1}\right)>\nu_{i+1}\left(Q_{\omega}\right), \nu_{i+1}(r) .
$$

This implies that $\nu\left(q Q_{i+1}\right)>\nu(r)$. Hence $\nu\left(Q_{\omega}\right)=\nu(r)$ and $\nu(r)=\infty$, then $r$ must be equal to 0 and $q=1$, since $\nu^{-1}(\infty)=\left(\mathbb{Q}_{\omega}\right)$. But $Q_{i+1} \neq Q_{\omega}$ and we have a contradiction.

By Proposition 5.3 we have $\nu_{i}=\mu_{i}$, hence

$$
\mu_{1}\left(Q_{\omega}\right)<\mu_{2}\left(Q_{\omega}\right)<\cdots<\mu\left(Q_{\omega}\right)
$$

We have a strictly increasing sequence in $\Gamma_{i_{1}}$, it most be unbouded in $\mathbb{R}$, hence $\mu\left(Q_{\omega}\right)=\infty$. This contradicts the fact that $\mu\left(Q_{\omega}\right)<\nu\left(Q_{\omega}\right)$.

Proposition 5.5. Either $\mu=\nu_{i_{0}-1}$ or $\mu=\mu_{i_{0}}<\nu_{i_{0}}$.

Proof. As in the proof of Proposition [5.3, we have $d_{i_{0}}(\nu) \leq d_{i_{0}}(\mu)$.

Suppose first that $\mu\left(Q_{i_{0}}\right)=\mu_{i_{0}-1}\left(Q_{i_{0}}\right)$. We will prove that in this case we have $\mu=\nu_{i_{0}-1}$.

Suppose, aiming for contradiction, that there exists $f \in K[y]$ such that $\mu(f)>\mu_{i_{0}-1}(f)$, and choose $f$ of minimal degree among all the polynomials having this property. Since

$$
d_{i_{0}}(\nu) \leq d_{i_{0}}(\mu)
$$

we have $\operatorname{deg}_{y}(f) \geq d_{i_{0}}(\nu)$.

Let $f=q Q_{i_{0}}+r$ be the Euclidean division of $f$ by $Q_{i_{0}}$. By the minimality of $\operatorname{deg} f$, we have $\mu(q)=\mu_{i_{0}-1}(q)$ and $\mu(r)=\mu_{i_{0}-1}(r)$.

We have $\nu_{i_{0}-1}(r)=\nu(r) \geq \inf \left\{\nu(f), \nu\left(q Q_{i_{0}}\right)\right\}>\inf \left\{\nu_{i_{0}-1}(f), \nu_{i_{0}-1}\left(q Q_{i_{0}}\right)\right\}$.

Hence $\nu_{i_{0}-1}(r)>\nu_{i_{0}-1}(f)=\nu_{i_{0}-1}\left(q Q_{i_{0}}\right)$.

But $\mu\left(q Q_{i_{0}}\right)=\nu_{i_{0}-1}\left(q Q_{i_{0}}\right)$ and $\mu(f)>\nu_{i_{0}-1}(f)$, hence $\mu(f)>\mu\left(q Q_{i_{0}}\right)=\mu(r)$, therefore $\nu_{i_{0}-1}\left(q Q_{i_{0}}\right)=\mu\left(q Q_{i_{0}}\right)=\mu(r)=\nu_{i_{0}-1}(r)$ which is a contradiction. 
Now suppose that $\mu\left(Q_{i_{0}}\right)>\mu_{i_{0}-1}\left(Q_{i_{0}}\right)$. We have $d_{i_{0}}(\mu)=d_{i_{0}}(\nu)$. We will prove that $\mu\left(Q_{i_{0}}\right)=\beta_{i_{0}}(\mu)$. Suppose that there exists a monic polynomial $Q$ such that $\operatorname{deg}_{y}(Q)=d_{i_{0}}(\mu)$, $\mu_{i_{0}-1}(Q)<\mu(Q)$ and $\mu(Q)<\mu\left(Q_{i_{0}}\right)$.

Write $Q=Q_{i_{0}}+g$ with $\operatorname{deg}_{y}(g)<d_{i_{0}}(\mu)$. We have $\mu\left(Q_{i_{0}}\right)>\mu(Q)=\mu(g)$. Therefore $\nu\left(Q_{i_{0}}\right)>\mu\left(Q_{i_{0}}\right)=\mu(g)=\nu(g)$. Hence $\nu\left(Q_{i_{0}}\right)>\nu(g)=\nu(Q)$, in particular,

$$
\beta_{i_{0}}(\nu)=\nu\left(Q_{i_{0}}\right)>\nu(Q)
$$

which contradicts the definition of $\beta_{i_{0}}(\nu)$.

We have $\beta_{i_{0}}(\mu)=\mu\left(Q_{i_{0}}\right), d_{i_{0}}(\mu)=d_{i_{0}}(\nu)$ and $\mu_{i_{0}}=\mu_{Q_{i_{0}}}$. It remains to prove that $\mu=\mu_{i_{0}}$.

Take any polynomial $f$ in $K[y]$. If $\operatorname{deg}_{y}(f)<d_{i_{0}}(\mu)$ then $\mu_{i_{0}}(f)=\mu(f)$. Suppose that $\operatorname{deg}_{y}(f) \geq d_{i_{0}}(\mu)$ and let $f=q Q_{i_{0}}+r$ be the Euclidean division of $f$ by $Q_{i_{0}}$.

If $\mu(f)>\mu_{i_{0}}(f)$ then $\mu(f)>\mu\left(q Q_{i_{0}}\right)=\mu(r)$. But $\nu(f) \geq \mu(f)$ and $\nu(r)=\mu(r)$, therefore $\nu(f)>\nu(r)=\nu\left(q Q_{i_{0}}\right)$. Then $\nu\left(q Q_{i_{0}}\right)=\mu\left(q Q_{i_{0}}\right)$ which is impossible, hence $\mu(f)=\mu_{i_{0}}(f)$.

Corollary 5.6. The valuations $\nu$ with $N(\nu)=\infty$ or $\left(N(\nu) \neq \infty\right.$ and $\left.\beta_{N(\nu)}(\nu)=\infty\right)$ are maximal elements of the set of valuations $\mu$ of $K(y)$ with $\mu(x)=1$.

From the preceding results we also deduce

Remark 5.7. 1. $N(\mu) \leq N(\nu)$ and $D(\mu) \leq D(\nu)$.

2. Either $\mu$ is the $y$-adic valuation with $\mu(y)<\nu(y)$, or there exists $i \in I(\nu)$ such that for each $j \leq i, \mu_{j}=\nu_{j}, I(\mu)=\{1, \ldots, i+1\},\left\{Q_{j}\right\}_{j \in I(\mu)}$ is a complete set of key polynomials for $\mu$ and $\mu=\left[\nu_{i}, Q_{i+1}, \mu\left(Q_{i+1}\right)\right]$.

3. $N(\mu)=N<\infty$ and $\mu$ and $\nu$ have the same sets of first $N$ key polynomials. More precisely, any set $\left\{Q_{i}\right\}_{i \in\{1, \ldots, N\}}$ of first $N$ key polynomials for $\mu$ is also a set of first $N$ key polynomials for $\nu$ and vice versa.

\subsection{Structure Theorems}

Theorem 5.8. Let $\tilde{\mu}$ and $\tilde{\nu}$ be two valuations in $\mathcal{V}$. Then there exists an infimum of $\tilde{\mu}$ and $\tilde{\nu}$ (that is, the greatest element that is less than or equal to $\tilde{\mu}$ and $\tilde{\nu}$ ) in the poset $\mathcal{V}$.

Proof. Fix local coordinates $x$ and $y$ such that $\tilde{\mu}(x)=\tilde{\nu}(x)=1$. Let $\mu$ and $\nu$ be the corresponding valuations on $k(x, y)$ under the correspondence in Theorem (3.12).

To prove the Theorem, we will prove that the infimum of $\mu$ and $\nu$ exists.

First we will define a valuation $\mu \wedge \nu$ and then prove that it is the infimum of $\mu$ and $\nu$.

Let $\left\{\nu_{i}\right\}_{i \in I(\nu)}$ and $\left\{\mu_{i}\right\}_{i \in I(\mu)}$ be the truncations associated to $\nu$ and $\mu$ respectively.

Suppose first that for each $i \in I(\nu) \cap I(\mu)$ we have $\nu_{i}=\mu_{i}$. If $I(\mu) \subseteq I(\nu)$ then $\mu \leq \nu$ and $\mu \wedge \nu=\mu$, otherwise, if $I(\mu) \subset I(\nu)$ then $\nu<\mu$ and $\mu \wedge \nu=\nu$. 
Now suppose that there exists $i \in I(\nu) \cap I(\mu)$ such that $\nu_{i} \neq \mu_{i}$. Let

$$
i_{0}=\inf \left\{i \in I(\nu) \cap I(\mu) \mid \nu_{i} \neq \mu_{i}\right\} .
$$

Suppose first that $\nu_{i_{0}}$ and $\mu_{i_{0}}$ are comparable. Without loss of generality, we may assume that $\mu_{i_{0}}<\nu_{i_{0}}$. In this case put $\mu \wedge \nu=\mu_{i_{0}}$. Clearly $\mu_{i_{0}} \leq \nu$ and $\mu_{i_{0}} \leq \nu$.

Let $\nu^{\prime}$ be a valuation of $k(x, y)$ such that $\nu^{\prime} \leq \mu$ and $\nu^{\prime} \leq \nu$. Since $\nu^{\prime} \leq \nu$, we have $\nu^{\prime}(x)=1$.

We know from Remark $5.7(1)$ that $N\left(\nu^{\prime}\right)<N(\mu)$ and $N\left(\nu^{\prime}\right)<N(\nu)$. Let $\left\{\nu_{i}^{\prime}\right\}_{i \leq N\left(\nu^{\prime}\right)}$ be the truncations associated to $\nu^{\prime}$.

From Remark 5.7 (2) we know that for each $i<N\left(\nu^{\prime}\right)$ we have $\nu_{i}^{\prime}=\mu_{i}$ and $\nu_{i}^{\prime}=\nu_{i}$, therefore $N\left(\nu^{\prime}\right) \leq i_{0}$.

We have $\nu^{\prime}=\nu_{N\left(\nu^{\prime}\right)}^{\prime} \leq \mu_{N\left(\nu^{\prime}\right)} \leq \mu_{i_{0}}=\mu \wedge \nu$.

Next, suppose that $\nu_{i_{0}}$ and $\mu_{i_{0}}$ are not comparable. In particular, we have $i_{0}>1$ (since $\mu_{1}$ and $\nu_{1}$ are always comparable). Put $\mu \wedge \nu=\mu_{i_{0}-1}=\nu_{i_{0}-1}$. Choose $\nu^{\prime}$ as in the paragraph above and let $\left\{\nu_{i}^{\prime}\right\}_{i \leq N\left(\nu^{\prime}\right)}$ be the truncations associated to $\nu^{\prime}$. By Remark 5.7 (3) the valuations $\nu^{\prime}$ and $\nu$ have the same set of $N\left(\nu^{\prime}\right)$ key polynomials, and the valuations $\nu^{\prime}$ and $\mu$ have the same set of $N\left(\nu^{\prime}\right)$ key polynomials. Therefore if, $N\left(\nu^{\prime}\right)=i_{0}$, we would have $\nu_{i_{0}}=\left[\nu_{i_{0}-1}, Q_{i_{0}}, \beta_{i_{0}}(\nu)\right]$ and $\mu_{i_{0}}=\left[\mu_{i_{0}-1}, Q_{i_{0}}, \beta_{i_{0}}(\mu)\right]$. The latter two valuations are comparable, hence $N\left(\nu^{\prime}\right) \leq i_{0}-1$. We have $\nu^{\prime}=\nu_{N\left(\nu^{\prime}\right)}^{\prime} \leq \mu_{N\left(\nu^{\prime}\right)} \leq \mu_{i_{0}-1}=\mu \wedge \nu$.

Theorem 5.9. Let $\tilde{S}$ be a totally ordered convex subset of $\mathcal{V}$. Then $\tilde{S}$ has a majorant in $\mathcal{V}$.

Remark 5.10. A short proof of a more general version of this result - one for rings of arbitrary dimension - is given in Lemma 3.9 (i) of [13] using elementary properties.

Proof. Since we are searching for a majorant, we may assume that $\tilde{S}$ contains $\tilde{\nu}_{\mathfrak{m}}$. Since $\tilde{S}$ is totally ordered, we can fix local coordinates $x$ and $y$ such that $\tilde{\nu}(y) \geq \tilde{\nu}(x)=1$ for all $\tilde{\nu} \in \tilde{S}$.

By Theorem 3.12, there exists a totally ordered convex subset $S$ of the set of valuations over $k(x, y)$, satisfying $1=\nu(x) \leq \nu(y)$ for all $\nu \in S$, and such that $S$ contains $\nu_{\mathfrak{m}}$. Also by Theorem 3.12 the set $\tilde{S}$ has a majorant in $\mathcal{V}$ if and only if the set $S$ has a majorant in the set of valuations over $k(x, y)$, satisfying $1=\nu(x) \leq \nu(y)$.

By Corollary 5.6, if $S$ contains an element $\nu$ with $N(\nu)=\infty$ or it contains an element $\nu$ with $\beta_{N(\nu)}(\nu)=\infty$ then $S$ has a maximal element. Suppose that $S$ does not contain a maximal element.

By Remark $5.7(1), N(\nu)$ and $D(\nu)$ define increasing functions on $S$.

We claim that there exists an initial segment $I \subset \mathbb{N}$ and a set of monic polynomials $\left\{Q_{i}\right\}_{i \in I(S)}$ such that for every valuation $\nu \in S$ the set $\left\{Q_{i}\right\}_{i \in I(\nu)}$ is complete for $\nu$ (the fact that $I \subset \mathbb{N}$ follows from the fact that $S$ does not contain a maximal element).

Indeed, take $\nu \in S, N \in \mathbb{N}$ and let $\left\{Q_{i}\right\}_{i \leq N}$ be a complete set of key polynomials for $\nu$. Let $\nu^{\prime} \in S$. If $\nu^{\prime}<\nu$, then by Remark 5.7 (3) the set $\left\{Q_{i}\right\}_{i \leq N\left(\nu^{\prime}\right)}$ is a complete set of key polynomials for $\nu^{\prime}$. Otherwise, if $\nu^{\prime}>\nu$, then, again by Remark [5.7 (3), we can add to $\left\{Q_{i}\right\}_{i \leq N}$ the key polynomials $\left\{Q_{i}\right\}_{N<i \leq N(\nu)}$ to obtain a complete set of key polynomials for $\nu^{\prime}$. 
Suppose first that $N(\nu)$ is bounded from above. In this case there exists $N \in \mathbb{N}$ with $I(S)=\{1, \ldots, N\}$, and a valuation $\nu \in S$ with $N(\nu)=N$.

The set $\left\{\beta_{N}(\nu) \mid \nu \in S, N(\nu)=N\right\}$ is bounded in $\overline{\mathbb{R}}$. Let $\bar{\beta}$ be a majorant for this set in $\overline{\mathbb{R}}$. If $N=1$, let $\mu$ be the $y$-adic valuation with $\mu(y)=\bar{\beta}$. Otherwise, if $N>1$, let $\mu=\left[\nu_{i-1}, Q_{N}, \bar{\beta}\right]$. Then $\mu$ is a majorant for $S$.

Now suppose that $N(\nu)$ is unbounded in $\mathbb{N}$, that is, $I(S)=\mathbb{N}$.

We have $D(\nu)<\infty$ for all $\nu \in S$ since $S$ does not contain a maximal element. Consider the set $D(S)=\{D(\nu) \mid \nu \in S\}$. Again, we have two cases, either $D(S)$ has a maximal element $D$, or it is unbounded in $\mathbb{N}$.

Suppose first that $D(S)$ is unbounded in $\mathbb{N}$. For each $f \in K[y]$, put

$$
\mu(f):=\max \{\nu(f) \mid \nu \in S\} .
$$

Note first that this maximum is well defined. Indeed, let $f \in K[y]$. Let $\nu \in S$ with

$$
D(\nu)>\operatorname{deg}_{y}(f) .
$$

For every $\nu^{\prime} \in S$ with $\nu \leq \nu^{\prime}$ we have $\nu(f)=\nu^{\prime}(f)$.

It is not difficult to verify that $\mu$ is a valuation on $k(x, y)$ and that $\mu$ is a majorant for $S$.

Now suppose that $D(S)$ has a maximal element $D$. There exists a cofinal sequence $\left\{\nu_{i}\right\}_{i \in I(S)}$ of valuations in $S$ with $\nu_{i}=\left[\nu_{i-1}, Q_{i}, \nu_{i}\left(Q_{i}\right)\right]$ for each $i>1$. Therefore, if we write $\beta_{i}=\nu_{i}\left(Q_{i}\right)$, the value group $\Gamma_{i}$ of $\nu_{i}$ is $\beta_{0} \mathbb{Z}+\cdots+\beta_{i} \mathbb{Z}$, with $\beta_{i} \in \mathbb{Q}$, by Proposition 4.6.

We claim that for every $f \in K[y]$, if there exists $i \in I(S)$ with $\nu_{i}(f)=\nu_{i+1}(f)$ then $\nu_{j}(f)=\nu_{i}(f)$ for all $j \in I(S), j \geq i$.

Indeed, let $i \in I(S)$ be such that $\nu_{i}(f)=\nu_{i+1}(f)$. By construction, we have

$$
\nu_{i}\left(Q_{i+1}\right)<\nu_{i+1}\left(Q_{i+1}\right)=\nu_{j}\left(Q_{i+1}\right) \quad \text { for all } j>i .
$$

Now let $f=q Q_{i+1}+r$ be the Euclidean division of $f$ by $Q_{i+1}$. Since $\nu_{i+1}(f)=\nu_{i}(f)$, we have $\nu_{i+1}\left(q Q_{i+1}\right)>\nu_{i+1}(f)=\nu_{i}(r)$. Now for all $j>i$ we have

$$
\nu_{j}\left(q Q_{i+1}\right) \geq \nu_{i+1}\left(Q_{i+1}\right)>\nu_{i}(r)=\nu_{j}(r) .
$$

Therefore $\nu_{j}(f)$ must be equal to $\nu_{j}(r)=\nu_{i}(r)=\nu_{i}(f)$.

If for all $f \in K[y]$ there exists $i \in I(S)$ with $\nu_{i}(f)=\nu_{i+1}(f)$, we put

$$
\mu(f):=\max _{i \in I(S)}\left\{\nu_{i}(f)\right\} .
$$

Otherwise, if there exists $f \in K[y]$ with

$$
\nu_{i}(f)<\nu_{i+1}(f) \quad \text { for all } i \in I(S),
$$

take $f$ monic of minimal degree, satisfying (5.2). We have $\operatorname{deg}_{y}(f)>D$ by definition of the polynomials $Q_{i}$ and the valuations $\nu_{i}$. Put $\mu(f)=\infty$. For a polynomials $g \in K[y]$, let $g=q f+r$ be the Euclidean division of $g$ by $f$, and put $\mu(g)=\max _{i \in I(S)}\left\{\nu_{i}(r)\right\}$. Then $\mu$ is a valuation of $k(x, y)$ which is a majorant for $S$. 


\section{Nonmetric Tree Structure on $\mathcal{V}$}

We will now define rooted non-metric trees.

Definition 6.1. A rooted non-metric tree is a poset $(\mathcal{T}, \leq)$ such that:

(T1) Every set of the form $I_{\tau}=\{\sigma \in \mathcal{T} \mid \sigma \leq \tau\}$ is isomorphic (as an ordered set) to a real interval.

(T2) Every totally ordered convex subset of $\mathcal{T}$ is isomorphic to a real interval.

(T3) Every non-empty subset $S$ of $\mathcal{T}$ has an infimum in $\mathcal{T}$.

Let us consider the following special case of the condition (T3):

$\left(\mathrm{T} 3^{\prime}\right)$ There exists a (unique) smallest element $\tau_{0} \in \mathcal{T}$.

Lemma 6.2. (Lemma 3.4 [13]) Under the conditions (T1) and (T3'), the following conditions are equivalent:

(T3) Every non-empty subset $S \subset \mathcal{T}$ has an infimum.

(T3") Given two elements $\tau, \sigma \in \mathcal{T}$, the set $\{\tau, \sigma\}$ has an infimum $\tau \wedge \sigma$.

Definition 6.3. A rooted nonmetric tree $\mathcal{T}$ is complete if every increasing sequence $\left\{\tau_{i}\right\}_{i \geq 1}$ in $\mathcal{T}$ has a majorant, that is, an element $\tau_{\infty}$, with $\tau_{i} \leq \tau_{\infty}$ for every $i$.

Theorem 6.4. The valuation space $\mathcal{V}$ is a complete nonmetric tree rooted at $\tilde{\nu}_{\mathfrak{m}}$.

Proof. (T3 $\left.{ }^{\prime}\right)$ It is clear that $(\mathcal{V}, \leq)$ is a partially ordered set with unique minimal element $\tilde{\nu}_{\mathfrak{m}}$.

(T1) Fix $\tilde{\nu}$ in $\mathcal{V}$, with $\tilde{\nu}>\nu_{\mathfrak{m}}$. We will show that the set $S=\left\{\tilde{\mu} \in \mathcal{V} \mid \nu_{\mathfrak{m}} \leq \tilde{\mu} \leq \tilde{\nu}\right\}$ is a totally ordered set isomorphic to an interval in $\overline{\mathbb{R}}_{+}$.

Choose local coordinates $x$ and $y$ such that $1=\tilde{\nu}(x) \leq \tilde{\nu}(y)$.

Let $\nu$ be the valuation of $k(x, y)$ corresponding to $\tilde{\nu}$ and let $\left\{Q_{i}\right\}_{i \in I(\nu)}$ be a complete sequence of key polynomials for $\nu$. The sequence $\frac{\beta_{i}(\nu)}{d_{i}(\nu)}$ is strictly increasing. If $I(\nu)$ has a maximal element $\ell$, put $I=\left[1, \frac{\beta_{\ell}(\nu)}{d_{\ell}(\nu)}\right] \subset \overline{\mathbb{R}}$. Otherwise, put $I=[1, \infty) \subset \overline{\mathbb{R}}$. We will prove that $S$ is isomorphic to $I$ as an ordered set.

To each $t \in I$ we will associate a valuation $\tilde{\nu}_{t}$ in $S$.

Let $t \in I$. If $t=1$, put $\tilde{\nu}_{t}=\tilde{\nu}_{\mathfrak{m}}$. If $I(\nu)$ has a maximal element $\ell$ and $t=\frac{\beta_{\ell}(\nu)}{d_{\ell}(\nu)}$, put $\tilde{\nu}_{t}=\tilde{\nu}$.

Now suppose that $1<t<\frac{\beta_{\ell}(\nu)}{d_{\ell}(\nu)}$. There exists a unique element $u \in I(\nu)$ such that $\frac{\beta_{u-1}(\nu)}{d_{u-1}(\nu)}<t \leq \frac{\beta_{u}(\nu)}{d_{u}(\nu)}$. Let $\nu_{t}:=\left[\mu_{u-1}, Q_{u}, t d_{u}\right]$ and $\tilde{\nu}_{t}$ the corresponding valuation in $\mathcal{V}$. That the resulting map is a bijection follows from Remark 5.7 .

(T2) By Theorem [5.9, every totally ordered convex subset $\tilde{S}$ of $\mathcal{T}$ has a majorant in $\mathcal{T}$. With $\left(\mathrm{T} 3^{\prime}\right)$ and (T1) this proves (T2). This also proves that $\mathcal{T}$ is complete. 
(T3) is an immediate consequence of Theorem 5.8 and Lemma 6.2 .

Remark 6.5. Let $(R, \mathfrak{m}, k)$ and $\left(R^{\prime}, \mathfrak{m}^{\prime}, k^{\prime}\right)$ be two regular two-dimensional local rings such that the residue fields $k$ and $k^{\prime}$ have the same cardinality. Let $\iota: k \cong k^{\prime}$ be a bijection between the two fields (as sets, that is, $\iota$ need not be a homomorphism of fields). Using the results of this paper it can be shown that $\iota$ induces a homeomorphism of the respective valuative trees, associated to $R$ and $R^{\prime}$. Thus, up to homeomorphism, a valuative tree associated to a regular local ring $(R, k)$ depends only on the cardinality of the residue field $k$.

\section{References}

[1] S. Abhyankar, On the valuations centered in a local domain, Amer. J. Math. 78 (1956), 321-348.

[2] N. Bourbaki, Algèbre Commutative, Chapitres 5 à 7, Masson, 1985.

[3] J. Decaup, M. Spivakovsky, W. Mahboub, Abstract key polynomials and comparison theorems with the key polynomials of Mac Lane - Vaquie. arXiv: 1611.06392v1 [math.AC].

[4] C. Favre and M. Jonsson, The Valuative Tree. Lec. Notes in Math. 1853. Springer-Verlag. (2004).

[5] A. Granja, The valuative tree of a two-dimensional regular local ring. Math. Res. Lett., 14, (2004), 19-34.

[6] F. J. Herrera Govantes, M. A. Olalla Acosta, M. Spivakovsky, Valuations in algebraic field extensions, Journal of Algebra, Volume 312, Issue 2 (2007), pages 1033-1074.

[7] F. J. Herrera Govantes, W. Mahboub, M. A. Olalla Acosta, M. Spivakovsky, Key polynomials for simple extensions of valued fields. arXiv:1406.0657v3 [math.AG].

[8] O. Kashcheyeva, Constructing examples of semigroups of valuations, Journal of Pure and Applied Algebra 220 (12) (2016), 3826-3860.

[9] S. MacLane, A construction for prime ideals as absolute values of an algebraic field, Duke Math. J. 2 (1936) 492-510.

[10] S. MacLane, A construction for absolute values in polynomial rings, Transactions of the AMS 40 (1936) 363-395.

[11] S. MacLane and O.F.G Schilling, Zero-dimensional branches of rank one on algebraic varieties, Ann. of Math.40 (1939) 507-520.

[12] W. Mahboub, Key Polynomials, Journal of Pure and Applied Algebra, 217 (6) (2013), 989-1006.

[13] J. Novacoski, Valuations centered at a two-dimensional regular local ring: Infima and Topologies, Valuation theory in interaction, 389-403, EMS Ser. Congr. Rep., Eur. Math. Soc., Zürich, 2014.

[14] J. Novacoski and M. Spivakovsky, Key polynoimals and pseudo-convergent sequences, Journal of Algebra 495 (2018), 199-219. 
[15] M. Spivakovsky, Valuations in Function Fields of Surfaces, American Journal of Mathematics (112) (1) (1990), 107-156.

[16] M. Vaquié, Valuations and local uniformization, Advanced Studies in Pure Mathematics 43, 2006, Singularity Theory and Its Applications, 477-527

[17] M. Vaquié, Extension d'une valuation. Trans. Amer. Math. Soc. 359 (2007), no. 7, 34393481.

[18] M. Vaquié, Michel, Famille admise associée à une valuation de K[x]. Singularités FrancoJaponaises, 391-428, Sémin. Congr., 10, Soc. Math. France, Paris, 2005.

[19] M. Vaquié, Famille admissible de valuations et défaut d'une extension, J. Algebra 311 (2007), no. 2, 859-876.

[20] O. Zariski, and P. Samuel, Commutative Algebra, Vol. II, New York/Heidelberg/Berlin (1960). 